\title{
Colors of a Second Earth: Estimating the fractional areas of ocean, land, and vegetation of Earth-like exoplanets
}

\author{
Yuka Fujii $^{1}$, Hajime Kawahara ${ }^{1}$, Yasushi Suto ${ }^{1,2,3}$, Atsushi Taruya ${ }^{1,2,4}$, Satoru Fukuda ${ }^{5}$, \\ Teruyuki Nakajima ${ }^{5}$, and Edwin L. Turner ${ }^{3,4}$ \\ yuka.fujii@utap.phys.s.u-tokyo.ac.jp
}

\begin{abstract}
Characterizing the surfaces of rocky exoplanets via their scattered light will be an essential challenge in investigating their habitability and the possible existence of life on their surfaces. We present a reconstruction method for fractional areas of different surface types from the colors of an Earth-like exoplanet. We create mock light curves for Earth without clouds using empirical data. These light curves are fitted to an isotropic scattering model consisting of four surface types: ocean, soil, snow, and vegetation. In an idealized situation where the photometric errors are only photon shot noise, we are able to reproduce the fractional areas of those components fairly well. The results offer some hope for detection of vegetation via the distinct spectral feature of photosynthesis on the Earth, known as the red edge. In our reconstruction method, Rayleigh scattering due to the atmosphere plays an important role, and for terrestrial exoplanets with an atmosphere similar to our Earth, it is possible to estimate the presence of oceans and an atmosphere simultaneously.
\end{abstract}

Subject headings: astrobiology — Earth — scattering — techniques: photometric

\footnotetext{
${ }^{1}$ Department of Physics, The University of Tokyo, Tokyo 113-0033, Japan

${ }^{2}$ Research Center for the Early Universe, Graduate School of Sciences, The University of Tokyo, Tokyo 113-0033, Japan

${ }^{3}$ Department of Astrophysical Sciences, Princeton University, Princeton, NJ 08544, USA

${ }^{4}$ Institute for the Physics and Mathematics of the Universe, The University of Tokyo, Kashiwa 277-8568, Japan

${ }^{5}$ Center of Climate System Research, The University of Tokyo, Kashiwa 277-8568, Japan
} 


\section{1. introduction}

A major milestone in exoplanet research will be the discovery of Earth-like planets. Indeed planets a few times as massive as the Earth, super-earths have already been discovered using the radial velocity method; for instance, $M_{\mathrm{p}} \sin i$ of GJ581e is estimated to $\sim 2 M_{\oplus}$ where $M_{\mathrm{p}}$ is the planetary mass and $i$ is the inclination of its orbit (Mayor et al. 2009). Microlensing and transit methods are well-suited for the detection of such low-mass planets; MOA-2007-BLG-192-Lb (Bennett et al. 2008) and CoRoT-7b (Queloz et al. 2009) are reported to have masses of $3.3_{-1.6}^{+4.9} M_{\oplus}$ and $4.8 \pm 0.8 M_{\oplus}$, respectively. The Kepler mission, launched on 2009 March 6, aims to detect a number of terrestrial planets with masses on the order of $M_{\oplus}$. Such low-mass planets are very likely to be rocky and may have bodies of liquid water on their surfaces if they orbit in Habitable Zone (HZ, e.g., Kasting et al. 1993, 2003) of the primary star. Their discovery will definitely trigger ever more serious investigations of techniques to search for signatures of life, or biomarkers.

The most conventional and extensively studied biomarkers are based on spectroscopic identification of molecular species in the planetary atmospheres, such as $\mathrm{O}_{2}$ and $\mathrm{O}_{3}$ (e.g., Leger et al. 1993; Des Marais et al. 2002). For the most favorable known exoplanetary systems, detection of such atmospheric absorption features is already within reach during the transit or the secondary eclipse; absorption features of $\mathrm{Na}, \mathrm{C}, \mathrm{O}$, and $\mathrm{H}$ in a hot Jupiter HD209458b (Charbonneau et al. 2002; Vidal-Madiar et al. 2003, 2004; Ballester et al. 2007) and $\mathrm{H}_{2} \mathrm{O}, \mathrm{CH}_{4}, \mathrm{CO}$, and $\mathrm{CO}_{2}$ in HD189733b (Tinetti et al. 2007; Swain et al. 2008, 2009) have been reported. Spectroscopy of non-transiting terrestrial planets orbiting in the HZ will likely require major and specialized observatories in space, but such facilities are being actively studied and evaluated by both NASA and ESA at present (e.g., Levine et al. 2009; Lawson et al. 2009; Darwin Mission Summary Status 2007).

An even more ambitious and direct approach to the search for life may be possible via multi-band photometry of terrestrial exoplanets that is observationally less demanding. In particular, the light scattered by the surface of a planet carries important information on properties of the surface. Ford et al. (2001) computed for the first time the diurnal variation of scattered light by the Earth observed at a distance of $10 \mathrm{pc}$. They showed that the fractional variation of light curves of the Earth is $10 \%-20 \%$, which indeed agrees with the result of Earthshine observations (e.g., Goode et al. 2001).

More importantly, these model light curves exhibited an increase in brightness due to the presence of vegetation around at $\lambda=750 \mathrm{~nm}$. This sharp increase of the albedo is known as the red edge. It is a fairly generic feature of vegetation on the Earth and is due to bio-pigments associated with photosynthesis. The red edge feature has been detected by Earthshine observations (Woolf et al. 2002; Arnold et al. 2002; Montañés-Rodríguez et al. 2006). It was sub- 
sequently discussed as a possible biomarker in extrasolar terrestrial planets by Seager et al. (2005) and Kiang et al. (2007a,b). Tinetti et al. (2006a,b) and Montañés-Rodríguez et al. (2006) performed more accurate simulations of the scattered radiation spectra of the Earth. They paid particular attention to the red edge, and discussed the detectability of its spectral feature.

Pallé et al. (2008) focused on the determination of the rotation period of an extrasolar terrestrial planet from the time variation of its scattered light. They concluded that the period can be reliably estimated in the presence of realistic partial cloud coverage of the planetary surface. They even found that the global circulation of the cloud pattern systematically modulates the estimate of the spin rotation period of the planet.

More recently, Cowan et al. (2009) approached the problem in a different and interesting manner. They performed a principal component analysis (PCA) on the real light curves of the Earth observed by the Extrasolar Planet Observation and Deep Impact Extended Investigation (EPOXI) mission. They identified two major eigenspectra that roughly correspond to ocean and land on the Earth and extracted a rough distribution of these components as a function of longitude. This was the first attempt to solve the inverse problem and to extract the surface properties from the observed data in a model-independent fashion. Williams \& Gaidos (2008) and Oakley \& Cash (2009) paid attention to the variation of scattered light according to the orbital motion of the planet. They pointed out that the reflectivity of ocean dramatically increases at a crescent phase and that the existence of the liquid water may be observationally inferred through the effect. Oakley \& Cash (2009) also suggested a possibility of reconstructing the land/ocean distribution along longitude using the gap between the reflectivity of ocean and that of land.

In this paper, we develop still another method to estimate the fractional areas of different surface types on extrasolar planets from multi-band photometry. We are particularly interested in terrestrial exoplanets in habitable zone and consider the detectability of vegetation using the red-edge feature. Our attempt is to reproduce the scattered light curves as a sum of the four surface types, i.e., ocean, soil, vegetation, and snow. While PCA attempts to extract only orthogonal eigenspectra in a model-independent manner, the correspondence with real surface types is not clear. Indeed, we would like to answer an ambitious, but welldefined, scientific question: "If we discover an Earth-like exoplanet in the near future, to what extent can we reconstruct the surface information, in particular the presence of vegetation observationally?". For that purpose, we intentionally and specifically adopt the major surface types of the Earth, including vegetation, into the analysis even though our method is thus inevitably model dependent. We discuss the feasibility of our method by applying it to mock scattered light curves of the cloudless Earth. We hope that our current results are rele- 
vant for a future TPF mission (Levine et al. 2009) such as the Occulting Ozone Observatory which aims at multi-band photometry of Earth-like planets (Kasdin et al. 2010).

The rest of this paper is organized as follows. Section 2 describes the generation of mock light curves of the Earth. The methodology, assumptions, and results of our inversion model to reconstruct the fractional areas are presented in Section 3 . We discuss the importance of the photometric band selection and comparison with PCA in Section 4. Finally we summarize the conclusion and discuss future directions of investigation in Section 5 .

\section{Mock scattered light curves of the Earth}

\subsection{Configuration of the Star-planet-observer System}

This section describes our computation method for generating mock light curves that are analyzed in the following section. Figure 11 illustrates the geometric configuration of the star-planet system adopted in this paper. The vectors $\mathbf{e}_{S}, \mathbf{e}_{\mathrm{R}}$, and $\mathbf{e}_{\mathrm{O}}$ denote the unit vectors from the center of the planet (E) toward the host star $(\mathrm{S})$, an arbitrary point on the surface $(\mathrm{R})$, and the observer $(\mathrm{O})$, respectively. A phase angle, $\alpha$, is defined as an angle between $\mathbf{e}_{\mathrm{S}}$ and $\mathbf{e}_{\mathrm{O}}$, which is fixed to $\alpha=\pi / 2$ in this paper, except where noted otherwise. The solar zenith angle, $\theta_{0}$, at $\mathrm{R}$ is an angle between $\mathbf{e}_{\mathrm{S}}$ and $\mathbf{e}_{\mathrm{R}}$, and the zenith angle of the observation, $\theta_{1}$, is that between $\mathbf{e}_{\mathrm{R}}$ and $\mathbf{e}_{\mathrm{O}}$. The azimuthal angle between incident direction and the direction of observation is represented by $\phi$ as shown in the right panel of Figure 1. In our fiducial configuration, the planet is located at the equinox (except in Section 3.4) and the observer is on the equatorial plane.

The incident ray from the host star toward the planet first passes through the atmosphere, which may scatter the ray. A fraction of the light reaches the planetary surface and is scattered there, and then returns to space passing through the scattering atmosphere once again. The scattering properties of the atmosphere depend on its composition and overall optical thickness, while those of the surface vary according to the details such as components, coarseness, moisture, the shape of surface, and so on. The wavelength-dependence of the synthetic scattered light therefore carries information about the atmosphere as well as the surface features. The fact that the illuminated and visible part of the planet gradually changes in time due to the motion of the planet (orbiting around its host star and spinning about its own axis) allows observers to scan the planetary surface in a fashion similar to global remote sensing of the Earth observation satellites. Thus, the variation of planetary light curves in different photometric bands allows mapping the planetary surface to some extent. 

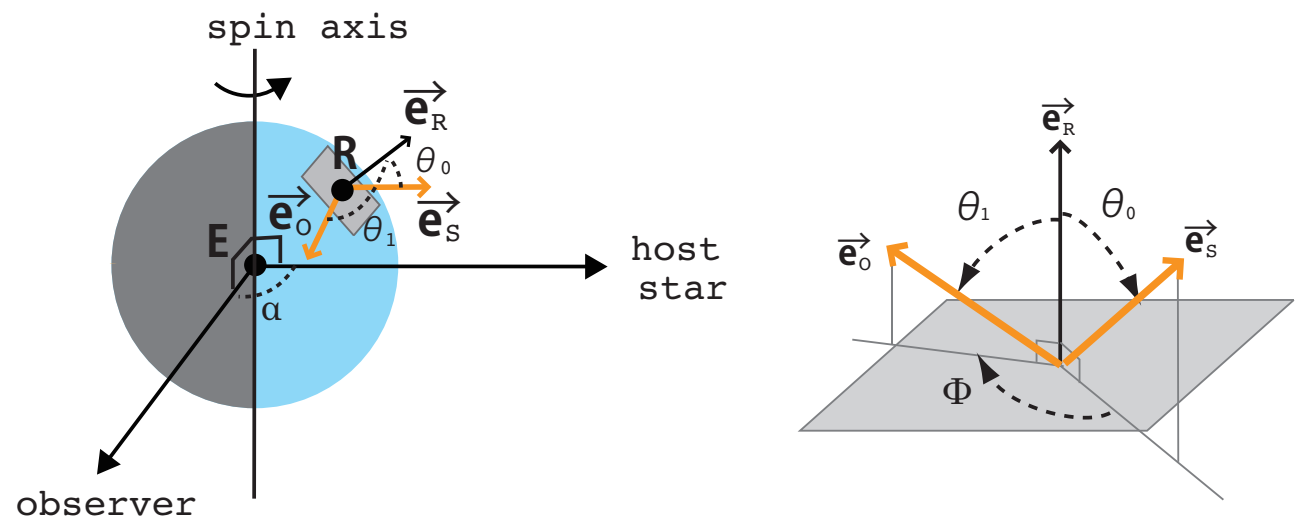

Fig. 1.- Schematic illustration of our fiducial configuration of the planetary system. The star is located at the equinox and the observer is in the equatorial plane. The phase angle $\alpha$ denotes the planet-centric angle between the host star and the observer, which is fixed to $\alpha=\pi / 2$. The unit vectors $\mathbf{e}_{\mathrm{S}}$ and $\mathbf{e}_{\mathrm{O}}$ go from the center of the planet (E) toward the host star $(\mathrm{S})$ and the observer $(\mathrm{O})$, respectively. The vector $\mathbf{e}_{\mathrm{R}}$ is a unit vector normal to the surface plane at $\mathrm{R}$. The solar zenith angle $\theta_{0}$, the zenith angle of the observation $\theta_{1}$, and the relative azimuthal angle $\phi$ are defined at each point $\mathrm{R}$ as the left panel indicates. As mentioned in Section 2.2, the illuminated pixels satisfy $\mathbf{e}_{\mathrm{S}} \cdot \mathbf{e}_{\mathrm{R}}>0$, and visible pixels satisfy $\mathbf{e}_{\mathrm{O}} \cdot \mathbf{e}_{\mathrm{R}}>0$.

\subsection{Model and Assumptions}

To generate synthetic scattered light curves, we approximate the planetary sphere by polygons of $2^{\circ} .5 \times 2^{\circ} .5$ "pixels." The angles $\theta_{0}, \theta_{1}$, and $\phi$ are defined at the center of each pixel. In general, the radiance $L\left(\theta_{0}, \theta_{1}, \phi ; \lambda\right)\left(\mathrm{W} \mathrm{str}^{-1} \mathrm{~m}^{-2} \mu \mathrm{m}^{-1}\right)$ of each pixel is expressed as

$$
L\left(\theta_{0}, \theta_{1}, \phi ; \lambda\right)=F_{*}(\lambda) \cos \theta_{0} f\left(\theta_{0}, \theta_{1}, \phi ; \lambda\right),
$$

where $F_{*}(\lambda)\left(\mathrm{W} \mathrm{m}^{-2} \mu \mathrm{m}^{-1}\right)$ is the incident flux at wavelength $\lambda$, and $f\left(\theta_{0}, \theta_{1}, \phi ; \lambda\right)\left(\operatorname{str}^{-1}\right)$ characterizes the scattering property of the surface and is conventionally referred to as the bidirectional reflectance distribution function (BRDF). The intensity of the total scattered light of a planet, $I(\lambda)\left(\mathrm{W} \operatorname{str}^{-1} \mu \mathrm{m}^{-1}\right)$, is obtained by integrating $L$ over the illuminated and visible region of the planetary surface $S$ :

$$
\begin{aligned}
I(\lambda) & =\int_{S} L\left(\theta_{0}, \theta_{1}, \phi ; \lambda\right) \cos \theta_{1} d S \\
& =\int_{S} F_{*}(\lambda) f\left(\theta_{0}, \theta_{1}, \phi ; \lambda\right) \cos \theta_{0} \cos \theta_{1} d S \\
& =F_{*}(\lambda) R_{\mathrm{p}}^{2} \int_{s} f\left(\theta_{0}, \theta_{1}, \phi ; \lambda\right) \cos \theta_{0} \cos \theta_{1} d s
\end{aligned}
$$


where $R_{\mathrm{p}}$ is the radius of the planet, $d s \equiv d S / R_{\mathrm{p}}^{2}$ is a normalized area element, and the illuminated and visible area $S$ is defined as a set of pixels that satisfy $\mathbf{e}_{\mathrm{S}} \cdot \mathbf{e}_{\mathrm{R}} \geq 0$ and $\mathbf{e}_{\mathrm{O}} \cdot \mathbf{e}_{\mathrm{R}} \geq 0$ simultaneously. Since the scattering property of a particular position on the surface gradually changes as the planet spins, $I(\lambda)$ varies as a function of time. Equation (2) is a key formula in computing the synthetic scattered light curve.

The evaluation of Equation (2) requires three inputs: $R_{\mathrm{p}}, F_{*}(\lambda)$ and $f\left(\theta_{0}, \theta_{1}, \phi ; \lambda\right)$. We approximate the incident flux $F_{*}(\lambda)$ from the star by a black-body spectrum:

$$
F_{*}(\lambda)=\frac{2 \pi h c^{2} R_{*}^{2}}{\lambda^{5}} \frac{1}{\exp \left(h c / \lambda k_{B} T_{*}\right)-1} \frac{1}{d^{2}}
$$

where $h$ is the Planck constant, $c$ is the speed of light, $k_{B}$ is the Boltzmann constant, $R_{*}$ and $T_{*}$ are the radius and the surface temperature of the star, and $d$ is the distance between the star and the planet. In this paper, we assume the Sun-Earth system, and therefore we set $R_{\mathrm{p}}=R_{\oplus}=6.4 \times 10^{6} \mathrm{~m}, T_{*}=T_{\odot}=5800 \mathrm{~K}, R_{*}=R_{\odot}=7.0 \times 10^{8} \mathrm{~m}$, and $d=1 \mathrm{AU}$.

We adopt a single scattering approximation for the interaction between the atmosphere and the underlying surface, i.e., the light is scattered once at most either in the atmosphere or at the surface and all multi-scattering processes are ignored. Then, the radiance of each pixel can be written as a linear combination of those associated with the surface and the atmosphere:

$$
L\left(\theta_{0}, \theta_{1}, \phi ; \lambda\right)=L_{\text {atm }}\left(\theta_{0}, \theta_{1}, \phi ; \lambda\right)+L_{\text {surf }}\left(\theta_{0}, \theta_{1}, \phi ; \lambda\right),
$$

or equivalently in terms of the BRDF (Equation (1)),

$$
f\left(\theta_{0}, \theta_{1}, \phi ; \lambda\right)=f_{\text {atm }}\left(\theta_{0}, \theta_{1}, \phi ; \lambda\right)+f_{\text {surf }}\left(\theta_{0}, \theta_{1}, \phi ; \lambda\right) .
$$

The specific models for $f_{\text {atm }}$ and $f_{\text {surf }}$ are described below.

\subsection{1. $f_{\mathrm{atm}}$ - Scattering by Atmosphere}

For scattering in the atmosphere represented by $f_{\text {atm }}$, we consider Rayleigh scattering alone, and ignore the effects of clouds, aerosols, and molecular absorption, which will be discussed elsewhere. We use a plane-parallel approximation locally for each pixel because our pixel size of $2.5^{\circ} \times 2.5^{\circ}$ is sufficiently small that one can reasonably approximate the overall sphere by a polygon consisting of such pixels. While this approximation is not valid for pixels near the edge of the illuminated and visible area, the fractional area of the region is small and our results are not changed significantly. 
Under the single-scattering approximation, the radiance from the atmosphere, $L_{\mathrm{atm}}$, is given by

$$
\begin{gathered}
L_{\mathrm{atm}}\left(\theta_{0}, \theta_{1}, \phi ; \lambda\right)=F_{*} \int_{0}^{\tau} \omega \Psi(\Theta) \exp \left\{-\tau^{\prime}\left(\frac{1}{\cos \theta_{0}}+\frac{1}{\cos \theta_{1}}\right)\right\} \frac{d \tau^{\prime}}{\cos \theta_{1}} \\
=\frac{F_{*} \omega \Psi(\Theta)}{\cos \theta_{1}} \frac{1}{1 / \cos \theta_{0}+1 / \cos \theta_{1}}\left[1-\exp \left\{-\tau\left(\frac{1}{\cos \theta_{0}}+\frac{1}{\cos \theta_{1}}\right)\right\}\right]
\end{gathered}
$$

or

$$
f_{\text {atm }}\left(\theta_{0}, \theta_{1}, \phi ; \lambda\right)=\frac{\omega \Psi(\Theta)}{\cos \theta_{0}+\cos \theta_{1}}\left[1-\exp \left\{-\tau(\lambda)\left(\frac{1}{\cos \theta_{0}}+\frac{1}{\cos \theta_{1}}\right)\right\}\right] .
$$

In the above expressions, $\omega$ is a single scattering albedo that is the ratio of scattering efficiency to total light attenuation (both scattering and absorption). In what follows, we ignore the effect of absorption and assume $\omega=1$.

The phase function for Rayleigh scattering, $\Psi(\Theta)$, is given by

$$
\Psi(\Theta)=\frac{3}{16 \pi}\left(1+\cos ^{2} \Theta\right)
$$

where $\Theta$ is the angle between the incident and scattered directions:

$$
\cos \Theta=\cos \theta_{0} \cos \theta_{1}+\sin \theta_{0} \sin \theta_{1} \cos \phi .
$$

The optical depth for Rayleigh scattering, $\tau$, is empirically given for the atmosphere of the Earth (e.g., Fröhlich and Shaw 1980; Young 1980):

$$
\tau=0.00864\left(\frac{P}{1013.25 \mathrm{mbar}}\right) \tilde{\lambda}^{-(3.916+0.074 \tilde{\lambda}+0.05 / \tilde{\lambda})} \propto \tilde{\lambda}^{-4},
$$

where $\tilde{\lambda}$ is wavelength in units of $1 \mu \mathrm{m}$, and $P$ is the pressure of the atmosphere at the base and we set $P=1013.25$ mbar everywhere for simplicity. The fact that $\tau$ is much less than unity in the visible and the near-infrared bands justifies the single-scattering approximation, that is adopted in this paper.

\subsection{2. $f_{\mathrm{surf}}$-Scattering by Surface}

The radiance of the surface, $L_{\text {surf }}$, is affected by atmospheric scattering as well, and is calculated by

$$
L_{\text {surf }}\left(\theta_{0}, \theta_{1}, \phi ; \lambda\right)=F_{*}(\lambda) \cos \theta_{0} f_{\text {surf }}\left(\theta_{0}, \theta_{1}, \phi ; \lambda\right),
$$

where

$$
f_{\text {surf }}\left(\theta_{0}, \theta_{1}, \phi ; \lambda\right)=C_{\text {atm }}\left(\theta_{0}, \theta_{1}, \phi ; \lambda\right) f_{\text {surf }, 0}\left(\theta_{0}, \theta_{1}, \phi ; \lambda\right),
$$


$f_{\text {surf, } 0}$ is the BRDF of the surface itself, and $C_{\text {atm }}$ describes attenuation due to atmospheric scattering before and after the ray reaches the surface:

$$
C_{\text {atm }}=\exp \left\{-\tau(\lambda)\left(\frac{1}{\cos \theta_{0}}+\frac{1}{\cos \theta_{1}}\right)\right\} .
$$

We consider the contributions of land, $f_{\text {land, } 0}$, and ocean, $f_{\text {ocean, } 0}$, in Section 2.2 .3 and Section 2.2.4, respectively.

\subsection{3. $f_{\text {land, } 0}-$ Scattering by Land}

Previous authors (Ford et al. 2001; Tinetti et al. 2006a, b); Montañés-Rodríguez et al. 2006; Pallé et al. 2008; Oakley \& Cash 2009) first classified the pixels on the surface into different surface types, and then computed the scattering due to each pixel according to its surface type. We do not apply any such classification, but rather use a parametrized BRDF for each pixel. More specifically, we adopt the model of MODerate resolution Imaging Spectroradiometer (MODIS; Salomonson et al. (1989)), an instrument on board the EOS/TERRA and AQUA satellites. The MODIS team processed the data using the Rossi-Li model for the BRDF, and assigned wavelength-dependent coefficients in this model to each observed pixel. The Rossi-Li model is one of semi-empirical kernel-based BRDF models and has been applied widely (e.g., Lucht et al. 2000). It consists of three terms:

$$
f_{\text {land }(\mathrm{RL})}\left(\theta_{0}, \theta_{1}, \phi ; \lambda\right)=f_{\text {iso }}(\lambda)+f_{\text {vol }}(\lambda) K_{\text {vol }}\left(\theta_{0}, \theta_{1}, \phi\right)+f_{\text {geo }}(\lambda) K_{\text {geo }}\left(\theta_{0}, \theta_{1}, \phi\right) .
$$

The first term represents the isotropic component. The second term (the volume-scattering term) represents the effect of the finite thickness of the scattering body. The last term (the geometric-optical term) takes into account of the effect of shadow. The explicit expressions for $K_{\mathrm{vol}}$ and $K_{\text {geo }}$ are given in Appendix A,

In this paper, we adopt the three coefficients in the Rossi-Li model $\left(f_{\text {iso }}, f_{\text {vol }}, f_{\text {geo }}\right)$ on each $2.5^{\circ} \times 2.5^{\circ}$ pixel from the dataset "snow-free gap-filled MODIS BRDF Model Parameters." This dataset is a spatially and temporally averaged product derived from the $0^{\circ} .05$ resolution $\mathrm{BRDF} /$ albedo data (v004 MCD43C1)1 1 . We select the BRDF parameters evaluated at the central position of each $2.5^{\circ} \times 2.5^{\circ}$ pixel and employ the values to represent the pixel.

The upper three panels of Figure 2 show the different behaviors of the surface brightness for each term in the Rossi-Li model. The lower-left panel plots $f_{\text {land(RL) }}\left(\theta_{0}, \theta_{1}, \phi ; \lambda\right)$ for

\footnotetext{
${ }^{1}$ The data-set is available through the MODIS web page http://modis.gsfc.nasa.gov/
} 
$f_{\text {iso }}=0.236, f_{\text {vol }}=0.114$ and $f_{\text {geo }}=0.027$ (these values are obtained by averaging over the pixels corresponding to land). Note that $f_{\text {land(RL) }}$ computed from Equation (14) is not positive definite, and becomes negative for very small $\theta_{0}$ or $\theta_{1}$. Thus, we set $f_{\text {land(RL) }}$ to 0 wherever it becomes negative. The lower-right panel of Figure 2 shows the scattering surface brightness due to the ocean described below.

\subsection{4. $f_{\text {ocean, } 0}-$ Scattering by Ocean}

The data set "snow-free gap-filled MODIS BRDF Model Parameters" does not assign parameters $\left(f_{\text {iso }}, f_{\text {vol }}, f_{\text {geo }}\right)$ for ocean pixels nor for pixels around the polar region. Our current model regards those pixels as ocean. Thus, our model systematically underestimates the snow and/or ice areas around the poles. Those components actually vary significantly from season to season as well, which is not yet taken into account in our present model either.

Since the scattering pattern by a liquid is very different from that by land, the above Rossi-Li model (Equation (14)) is not relevant for oceans. Instead, we adopt the ocean BRDF model of Nakajima \& Tanaka (1983). We describe their model below. Further details are discussed in Appendix B.

Their model approximately computes the scattering of ocean by the sum of contributions from small facets (Appendix B and Figure [18). The facets are characterized by the angle of their normal directions $\theta_{n}$, and the scattering for each facet follows the simple Snell-Fresnel law. The distribution function $p\left(\theta_{n}\right)$ for the slope of those facets depends on the wind speed above the ocean (Equations $(\underline{\mathrm{B} 9})$ and $(\underline{\mathrm{B} 10})$ ). Thus, their BRDF model is expressed as

$$
f_{\text {ocean }}\left(\theta_{0}, \theta_{1}, \phi ; \lambda\right)=\frac{1}{4 \cos \theta_{0} \cos \theta_{1} \cos \theta_{n}^{*}} p\left(\theta_{n}^{*}\right) G\left(\theta_{0}, \theta_{1}, \phi, u_{10}\right) r\left(\theta_{0}, \theta_{1}, \theta_{n}^{*}, \phi, \tilde{m}\right),
$$

where $\theta_{n}^{*}$ indicates the direction of the wave slope responsible for the specular reflection, $u_{10}$ is the wind speed at $10 \mathrm{~m}$ height above the surface, and $\tilde{m}=\tilde{m}(\lambda)$ is the ratio of the refractive index of ocean to atmosphere. The term $r\left(\theta_{0}, \theta_{1}, \phi, \tilde{m}\right)$ stands for Fresnel's scattering coefficient, $p\left(\theta_{n}\right)$ is the density distribution function of the wave slope, and $G\left(\theta_{0}, \theta_{1}, \phi, u_{10}\right)$ represents the bidirectional shadowing effect.

Our simulation adopts $u_{10}=4 \mathrm{~ms}^{-1}$, which corresponds to the spatial average of the wind speed at $10 \mathrm{~m}$ above the ocean, which is the average of monthly wind speed 2 . The

\footnotetext{
${ }^{2}$ the NOAA-ESRL Physical Sciences Division, Boulder Colorado. http://www.cdc.noaa.gov/
} 
color of the ocean is affected by the scattering of light inside the ocean, but we neglect this process for simplicity. We plan to incorporate it in future work.

The surface brightness of ocean in this model is shown in the lower middle panel of Figure 2. The color contours clearly exhibit the remarkable increase in reflectance of the ocean near $\theta_{0}=\theta_{1}$ due to specular reflection. The strong specular reflection feature will be further discussed in Section 3.5.

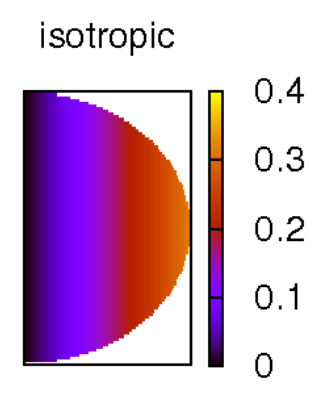

Rossi-Li

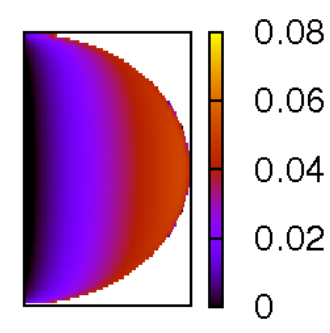

volume

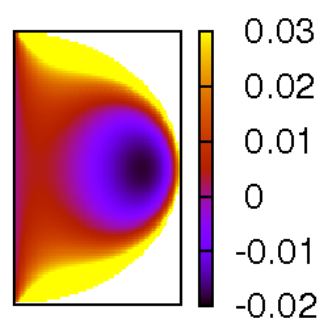

ocean

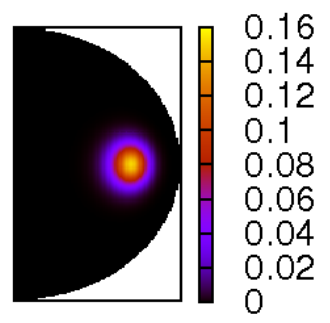

geometric

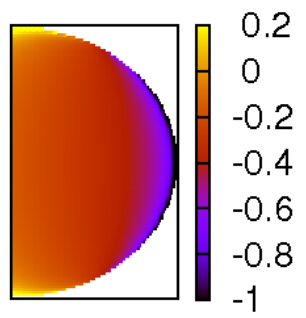

Rayleigh(SS)

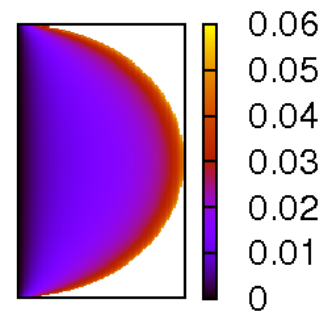

Fig. 2.- Surface brightness of the sunlit hemisphere of the planet seen at $\alpha=\pi / 2$ normalized by solar flux. The incident ray is coming from the right. The "isotropic" panel shows the surface brightness due to the isotropic term (assuming $f_{\text {iso }}=1$ ), the "volume" panel is due to the $f_{\mathrm{vol}} K_{\mathrm{vol}}$ term (assuming $f_{\mathrm{vol}}=1$ ), the "geometric" panel is due to the $f_{\text {geo }} K_{\text {geo }}$ term (assuming $f_{\text {geo }}=1$ ), and the "Rossi-Li" panel is due to the combination of the three terms, $f_{\mathrm{RL}}$, assuming $\left(f_{\text {iso }}, f_{\mathrm{vol}}, f_{\text {geo }}\right)=(0.236,0.114,0.027)$, a set of averaged values over the entire land surface. The "ocean" panel is based on the ocean BRDF model by Nakajima \& Tanaka (1983) with a wind velocity $4 \mathrm{~m} \mathrm{~s}^{-1}$. The "Rayleigh (SS)" panel is the surface brightness due to Rayleigh scattering of the atmosphere within a single-scattering (SS) and a flat atmospheric layer approximations, which are quantitatively not accurate at the edges, where either $\theta_{0}$ and $\theta_{1}$ are close to zero.

Adopting the above scattering model, we are now able to compute scattered light curves of a "Second Earth" (but without clouds). The planet rotates around its spin axis with a 
period of $24 \mathrm{hr}$. The parameters of our mock observations are listed in Table 1 .

We ignore the effect of the spin rotation during the exposure time $t_{\text {exp. }}$ The orbital motion of the planet is completely ignored during the observation period $n$. We employ the set of MODIS photometric bands on which our data for the land BRDF are based. These bands are 0.459-0.479 $\mu \mathrm{m}$ (band 1), 0.545-0.565 $\mu \mathrm{m}$ (band 2), 0.620-0.670 $\mu \mathrm{m}$ (band 3), 0.841-0.876 $\mu \mathrm{m}$ (band 4), 1.230-1.250 $\mu \mathrm{m}$ (band 5), 1.628-1.652 $\mu \mathrm{m}$ (band 6), and 2.105$2.155 \mu \mathrm{m}$ (band 7). In reality, we do not integrate over the band but simply calculate the scattering at the central wavelength of each band and multiply it by the band width.

In practice, the spin rotation period of the planet is unknown a priori, and we must first determine it from the observed light curves. Pallé et al. (2008) discussed how to infer the spin rotation period from the photometric variation and found that auto-correlation analysis of light curves can effectively determine the period. In this paper, therefore, we assume that the spin rotation period of the planet is precisely known, and fold the light curves accordingly.

\subsection{Results}

Figure 3 shows the simulated light curves in the seven bands. We consider a very idealized observational situation in which the light from the host star is completely blocked, and the photometric noise is due to the Poisson fluctuations in the observed photon counts from the planet alone:

$N \sim 840\left(\frac{I}{10^{15} \mathrm{~W} \mathrm{str}^{-1} \mu \mathrm{m}^{-1}}\right)\left(\frac{l}{10 \mathrm{pc}}\right)^{-2}\left(\frac{D}{2 \mathrm{~m}}\right)^{2}\left(\frac{t_{\text {exp }}}{1 \mathrm{hr}}\right)\left(\frac{n}{14 \text { days }}\right)\left(\frac{\lambda}{1 \mu \mathrm{m}}\right)\left(\frac{\Delta \lambda}{0.1 \mu \mathrm{m}}\right)$.

Table 1. Canonical Values of Parameters Assumed in Our Simulations of Photometric Light Curves.

\begin{tabular}{lcc}
\hline \hline \multicolumn{1}{c}{ Parameter } & Symbol & Value \\
\hline Planet-observer distance & $l$ & $10 \mathrm{pc}$ \\
Effective diameter of telescope & $D$ & $2 \mathrm{~m}$ \\
Exposure time & $t_{\exp }$ & $1 \mathrm{hr}$ \\
Observation period & $n$ & 14 days \\
\hline
\end{tabular}



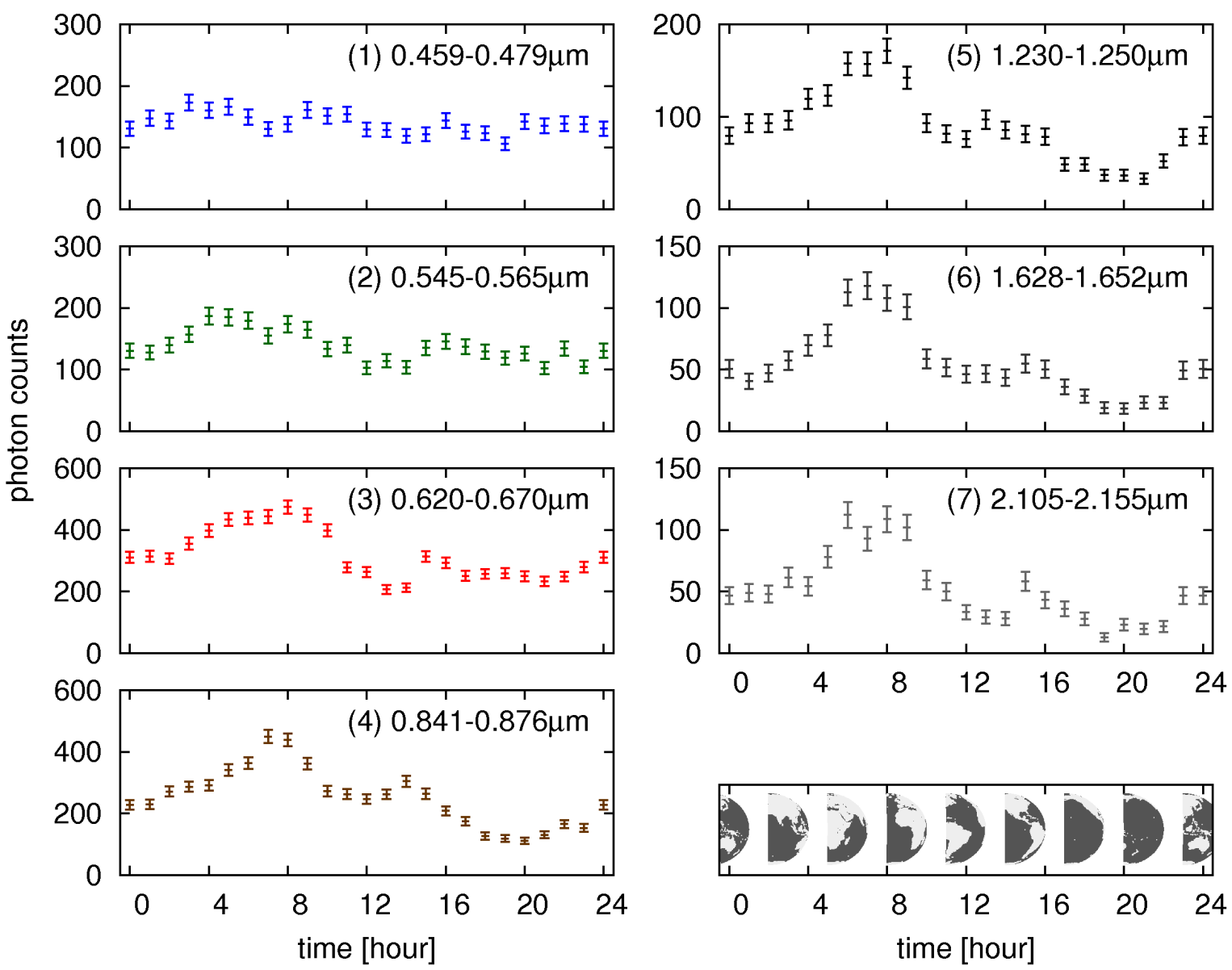

Fig. 3. - Light curves of the cloudless Earth at 0.459-0.479 $\mu \mathrm{m}$ (band 1), 0.545-0.565 $\mu \mathrm{m}$ (band 2), 0.620-0.670 $\mu \mathrm{m}$ (band 3), 0.841-0.876 $\mu \mathrm{m}$ (band 4), 1.230-1.250 $\mu \mathrm{m}$ (band 5), 1.628-1.652 $\mu \mathrm{m}$ (band 6) and 2.105-2.155 $\mu \mathrm{m}$ (band 7). The error bars come from photon shot noise only. The bottom right panel shows the snapshots of the Earth at corresponding epochs. The ocean is painted in black and the land is in white. The observer is located on celestial equator and half of the projected planetary surface is illuminated.

Here, the errors scale as $\sim \sqrt{\mathrm{N}}$. In reality, however, there are certainly many sources of errors such as contamination by the host star, zodiacal light and detector noise; furthermore the flux from the planet will be attenuated through the telescope optical instrument and detectors. However for this preliminary investigation, we consider this idealized observational situation and leave a more realistic treatment of errors to future studies.

Light curves at short wavelengths (especially in bands 1 and 2) in Figure 3 do not exhibit significant time variation because Rayleigh scattering by the atmosphere is dominant at 
shorter wavelengths and dilutes the variations of surface features. The atmosphere becomes significantly more transparent at longer wavelengths and the variations of light curves due to the inhomogeneous surface become appreciable. A comparison of the light curves with the snapshots in Figure 3 indicates that the three bumps at $t \sim 1,8$, and 14 hr indeed correspond to the Eurasian, African, and American continents. The highest peak shows up when the Sahara desert emerges. A dip at $t \sim 20 \mathrm{hr}$ occurs when the illuminated and visible part is covered with ocean, since the reflectivity of land is higher than that of ocean, especially at longer wavelengths. Another dip at $t \sim 13 \mathrm{hr}$ occurs when the South American continent replaces the specular reflection point. All these features are consistent with the light curve simulation of a cloudless Earth by Ford et al. (2001) and Oakley \& Cash (2009), and are essential in extracting information concerning the surface features of a planet from its light curves.
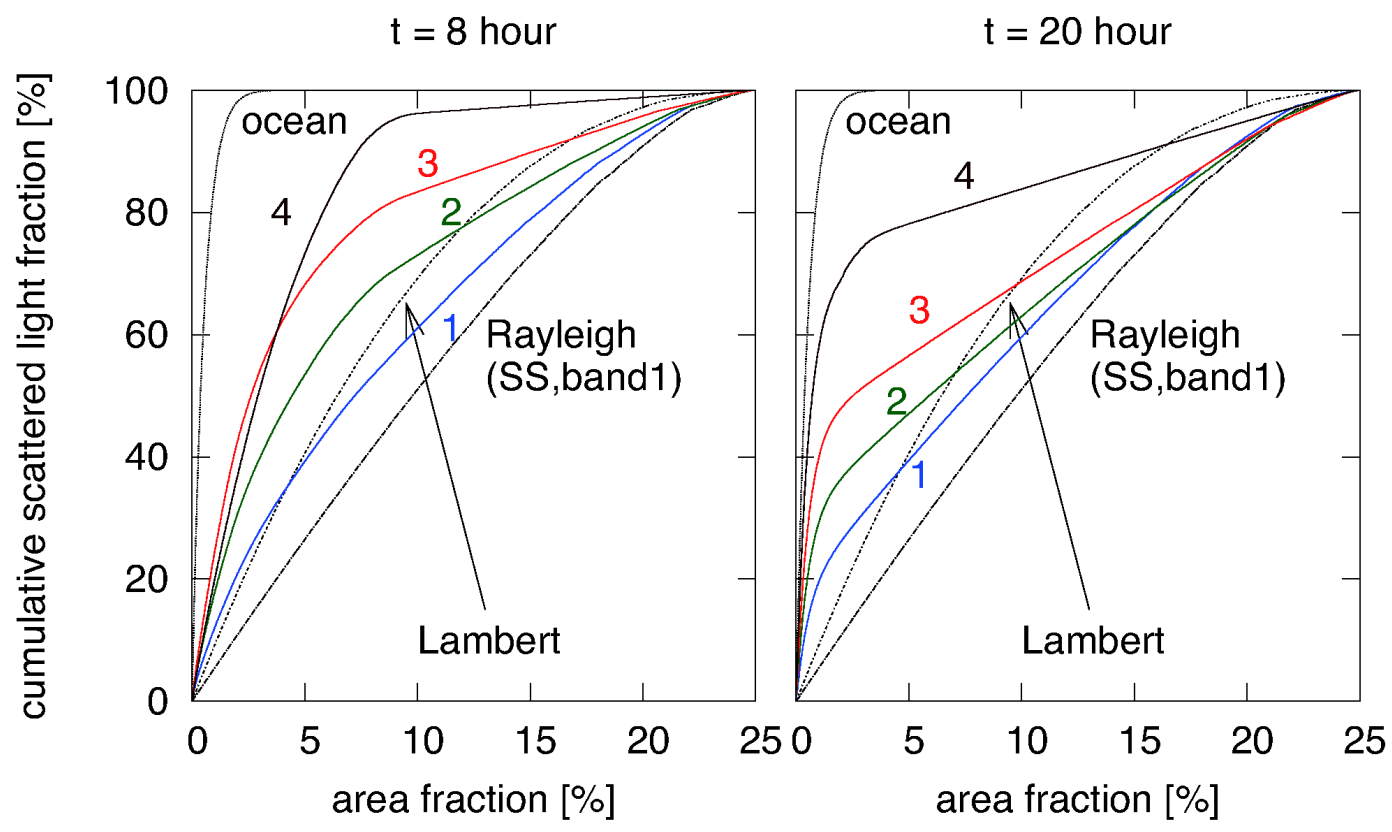

Fig. 4. - Scattering properties of different types of surfaces. Cumulative fraction of scattered light is plotted against the corresponding fractional area defined as responsible for the scattered light. The lines at $t=8,20 \mathrm{hr}$ are plotted with three references - ocean with wind velocity $u_{10}=4 \mathrm{~m} \mathrm{~s}^{-1}$, Rayleigh scattering and Lambertian. Different colors represent different bands as in Figure 3 (band 1: blue, band 2: green, band 3: red, band 4: brown). The light scattered by the ocean is very localized.

Figure 4 illustrates the degree of localization of the source of the observed flux from the planetary surface. According to the geometry of the system that we adopt here, a quarter of the planetary surface area is illuminated and visible to the observer. We sort all the 
pixels located in the illuminated and visible part of the surface according to the amount of scattered light per area, and compute the cumulative factional scattered light as a function of the corresponding fractional area. In Figure 4, if all the pixels contribute to the light equally, the resulting plot would be a straight line reaching $100 \%$ at the fractional area of $25 \%$. In reality, however, the plot is slightly curved even in the case of isotropic scattering (Lambertian) due to the curvature of the global surface (Figure 21). Since light from the ocean region comes from a very localized specular spot, the dotted line in Figure 4 is very steep at small fractional area - the ocean spot equivalent to $\sim 2 \%$ of the surface area is responsible for nearly the entire light from the ocean.

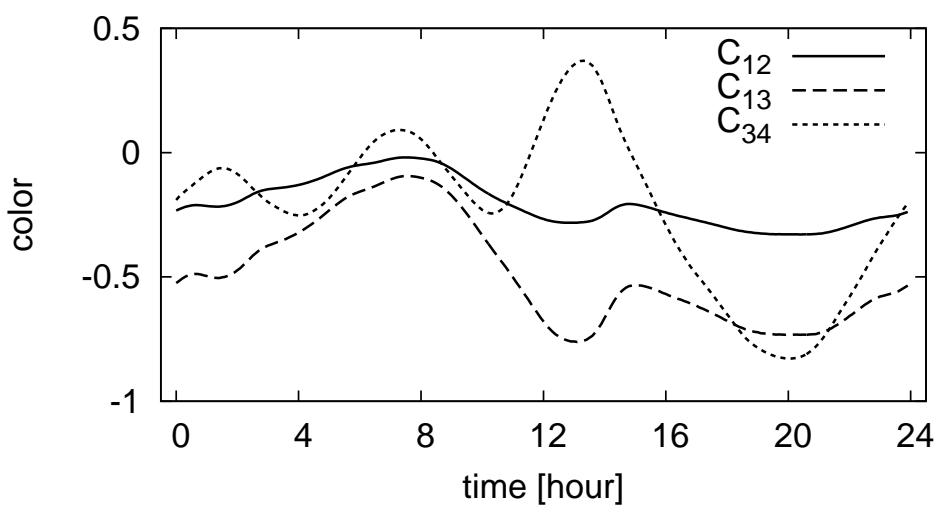

Fig. 5.- Variation of colors of Earth against time. Considered bands are 0.459-0.479 $\mu \mathrm{m}$ (band 1), 0.545-0.565 $\mu \mathrm{m}$ (band 2), 0.620-0.670 $\mu \mathrm{m}$ (band 3), and 0.841-0.876 $\mu \mathrm{m}$ (band 4). Here, "color" of band $a$ and $b$ is defined as $C_{a b} \equiv-2.5 \log \frac{I_{a}}{I_{b}}$ where $I_{a}$ and $I_{b}$ are the energy fluxes per wavelength in band $a$ and band $b$.

Essentially, the information that we utilize in reconstructing the fractional areas is color variations at different phases of the surface. To put it more clearly, we define the color between band $a$ and band $b$ as

$$
C_{a b} \equiv-2.5 \log \frac{I_{a}}{I_{b}}
$$

where $I_{a}$ and $I_{b}$ are the energy fluxes per wavelength in band $a$ and band $b$ (Equation (2)). Figure 5 shows time variations of $C_{12}, C_{13}$, and $C_{34}$. The trajectory on the $C_{13}-C_{34}$ plane is plotted in Figure 6 together with the typical colors of ocean, snow, vegetation, soil, and Rayleigh scattering which will be described in the next section and in Figure 7. The trajectory indicates that the illuminated and visible part of the surface is almost fully covered by ocean at $t=20 \mathrm{hr}$. The other tips at $t=8$ and $13 \mathrm{hr}$ correspond to the African continent with the Sahara desert and the South American continent with the Amazon forest, 
respectively. These color variations play a key role in estimating fractional areas of different surface types.

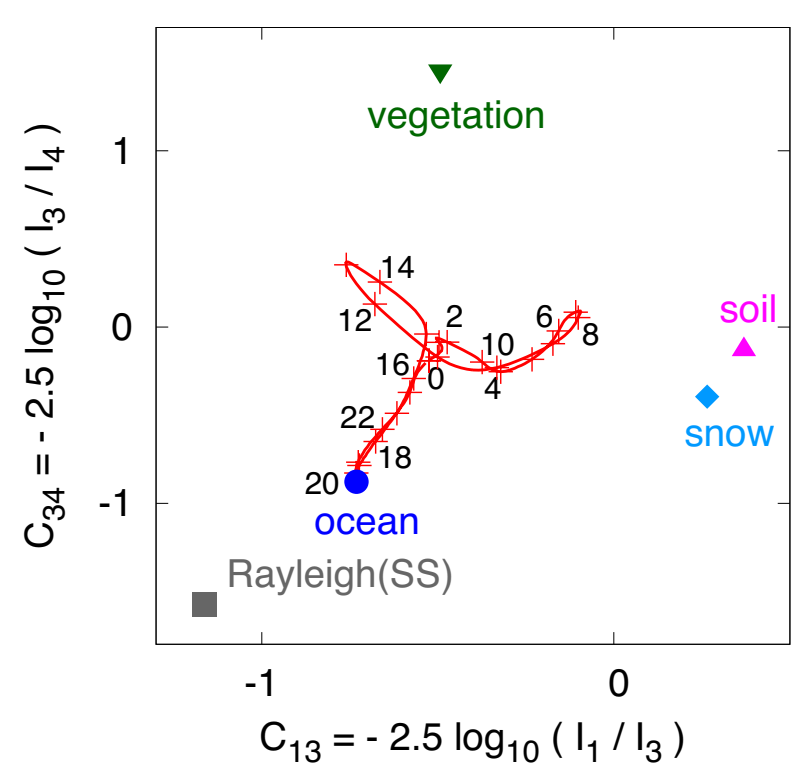

Fig. 6.- Noiseless light curve trajectory on a color-color diagram assuming a black-body spectrum as the incident flux. The "color" of band $a$ and $b$ is defined as $C_{a b} \equiv-2.5 \log \frac{I_{a}}{I_{b}}$ where $I_{a}$ and $I_{b}$ are the energy fluxes per wavelength in band $a$ and band $b$. The numbers denote the time (hours) corresponding to those in Figure 3. The symbols labeled by "ocean," "soil," "vegetation," "snow," and "Rayleigh (SS)" indicate the locations of the typical scattering colors of that surface type with atmospheric layer on it. Wavelength-dependent albedo of these five types are exhibited in Figure 7.

\section{Reconstruction of the fractional areas of surface types}

In this section, we describe our methodology for reconstructing the fractional areas of different surface types from multi-band photometry and present the results from the analysis of the mock light curves described in Section 2 .

\subsection{Inversion Method}

Our basic strategy for reconstructing the surface features of a planet is to fit the photometric light curves with an a priori model of planetary scattering. In doing so, we adopt 
two major simplifying assumptions. One is the Lambertian model (i.e., isotropic scattering) for the surface, and the other is that the surface consists solely of four different types (ocean, soil, vegetation, and snow) plus an atmosphere.

The Lambertian surface is one of the simplest models of scattering with constant radiance against any geometry of incident and scattered rays. The BRDF for a Lambertian surface is simply given as

$$
f_{k}\left(\theta_{0}, \theta_{1}, \phi ; \lambda\right)=f_{\text {iso } k}(\lambda)=\frac{a_{k}(\lambda)}{\pi}
$$

where the subscript $k$ denotes an index of the surface types and $a_{k}(\lambda)$ is a wavelengthdependent albedo of the $k$-th surface type.

The validity and limitation of our reconstruction method crucially depend on the number of different surface types that we consider. In practice, however, the limited information of color variations strongly restricts the number that can be uniquely determined by the analysis. Thus we consider only four types ( $k=$ ocean, soil, vegetation, and snow) that constitute the major components of the surface of the Earth.

This approach inevitably limits the generality of our model. Nevertheless we think it reasonable for the present purposes for several reasons. First, our Earth is currently the only known example of a habitable planet, and it is not unreasonable to assume that at least some habitable exoplanets have similar surface properties. Second, these four types represent very different albedos (Figure 7), which makes it easier to distinguish them in scattered light. Finally, the presence of ocean(s) on a planet is deeply related to the fundamental question of its habitability and the vegetation red edge can be regarded, if it exists at all, as a direct indication of the presence of life.

Under the conditions described above, Equation (2) at a given epoch $t_{i}$ reduces to

$$
\begin{aligned}
I(\lambda) & =F_{*}(\lambda) R_{\mathrm{p}}^{2} \int_{S} f_{\text {iso } k}(\lambda) \cos \theta_{0} \cos \theta_{1} d s \\
& =F_{*}(\lambda) R_{\mathrm{p}}^{2} \sum_{k}\left\{\frac{a_{k}(\lambda)}{\pi} \int_{s_{k}} \cos \theta_{0} \cos \theta_{1} d s\right\},
\end{aligned}
$$

where the integration is performed over $s_{k}=s_{k}\left(t_{i}\right)$ that is the area of the $k$-th surface type in the illuminated and visible area at $t_{i}$. Denoting each band by an index $j(=1,2 \ldots, 7)$, we discretize Equation (19) as

$$
I_{j}\left(t_{i}\right)=F_{* j} R_{\mathrm{p}}^{2} \int_{s} \cos \theta_{0} \cos \theta_{1} d s \sum_{k} D_{j k} A_{k}\left(t_{i}\right),
$$




$$
\begin{aligned}
D_{j k} & \equiv f_{\text {iso } j k}=\frac{a_{j k}}{\pi} \\
A_{k}\left(t_{i}\right) & \equiv \frac{\int_{s_{k}\left(t_{i}\right)} \cos \theta_{0} \cos \theta_{1} d s}{\int_{s} \cos \theta_{0} \cos \theta_{1} d s}
\end{aligned}
$$

where $A_{k}$ is the geometrically-weighted fractional area of the $k$-th surface type that we want to estimate, and $D_{j k}$ is normally referred to as the "design matrix" by statisticians.

In Equation (20), the incident flux $F_{* j}$ is calculated from the intensity of the host star and the distance between the star and the planet $d$, which is obtained once the orbit of the planet is determined. The integral $\int_{s} \cos \theta_{0} \cos \theta_{1} d s$ depends on the phase angle $\alpha$ alone (in our current configuration of the phase angle $\alpha=\pi / 2, \int_{s} \cos \theta_{0} \cos \theta_{1} d s=2 / 3$ ).

In order to estimate the weighted fractional area $A_{k}\left(t_{i}\right)$, we need to determine the design matrix, $D_{j k}$ from the albedo $a_{j k}$. We define the effective albedo of the $k$-th surface type, $a_{\text {eff } k}(\lambda)$, by setting it equal to the actual reflectivity when the whole surface is covered by that surface type. More specifically, it is computed as

$$
a_{\mathrm{eff} k}(\lambda) \equiv \pi \frac{\int_{s} f_{\text {model } k}\left(\theta_{0}, \theta_{1}, \phi ; \lambda\right) \cos \theta_{0} \cos \theta_{1} d s}{\int_{s} \cos \theta_{0} \cos \theta_{1} d s},
$$

where $f_{\text {model } k}$ is the model BRDF of the $k$-th surface type and the integrations are performed over the illuminated and visible area which, again, depends on the phase angle $\alpha$ alone. Then we define $a_{j k}$ of the $j$ th band from its central wavelength, $\lambda_{j}$, as

$$
a_{j k}=a_{\mathrm{eff} k}\left(\lambda_{j}\right)
$$

The model BRDF $f_{\text {model } k}$ in Equation (23) is calculated from

$$
f_{\text {model } k}\left(\theta_{0}, \theta_{1}, \phi ; \lambda\right)=f_{\text {atm }}\left(\theta_{0}, \theta_{1}, \phi ; \lambda\right)+C_{\text {atm }}\left(\theta_{0}, \theta_{1}, \phi ; \lambda\right) f_{\text {surf }, 0 k}\left(\theta_{0}, \theta_{1}, \phi ; \lambda\right),
$$

where $f_{\text {atm }}$ and $C_{\text {atm }}$ are given by Equations (7) and (13), respectively. In order to determine $f_{\text {surf }, 0 k}$ of the three land components ( $k=$ soil, vegetation and snow), we first assume that they are Lambertian with scattering spectra given by the ASTER spectral library 3 (Baldridge et al. 2008). Specifically, we adopt "Brown to dark brown sand (Entisol)," "Grass," and "Fine Snow" for soil, vegetation, and snow, respectively. Since the ASTER

\footnotetext{
${ }^{3}$ http://speclib.jpl.nasa.gov/
} 
spectral library offers data at discrete wavelengths, we linearly interpolate the data to obtain the $f_{\text {surf }, 0 k}$ suitable for our MODIS bands. For $f_{\text {surf }, 0 k}$ of ocean, we use Equation (15)). Figure 7 displays the effective albedo at $P=1013.25$ mbar for ocean, soil, vegetation, and snow as a function of wavelength $\lambda$. The dashed lines are the albedo curves in the absence of an atmosphere. The black solid line is the effective albedo due to atmospheric Rayleigh scattering. The primary effect of Rayleigh scattering is to add a very blue continuum to every pixel's contribution to the total light, but in our single-scattering approximation it also reduces the amount of light reaching the surface. In Figure 7, this is most clearly visible in the blue spectral region for the snow component.

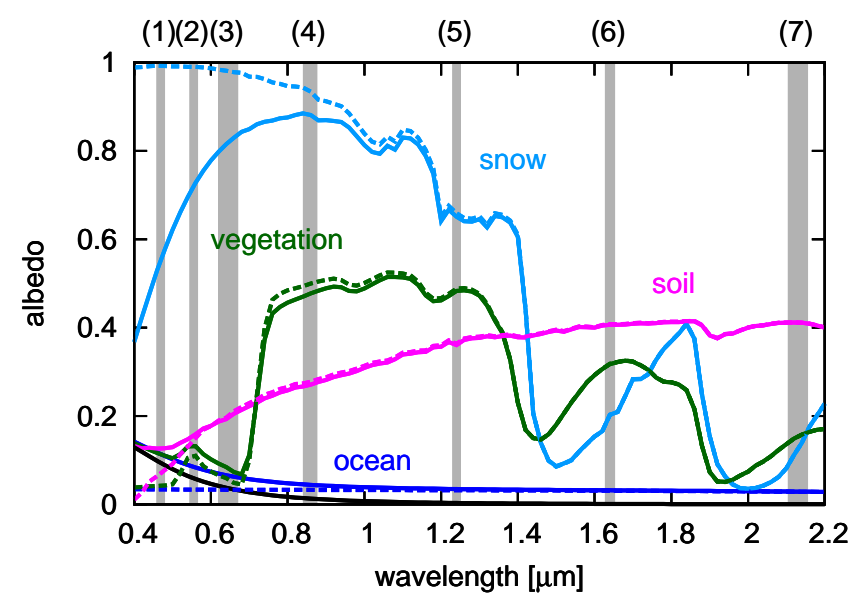

Fig. 7.- Wavelength-dependent effective albedos of ocean (blue), soil (magenta), vegetation (green), snow (cyan), and atmosphere with Rayleigh scattering alone (black). The solid lines show the effective albedo with Earth-like atmosphere, while the dashed lines show the effective albedo without an atmosphere. Shaded regions correspond to the MODIS bands. The numbers at the top are the labels of the different photometric bands.

\subsection{Fitting}

Now we apply the above methodology to the mock light curves (Section 2). We determine the best-fit values of $A_{j}\left(t_{i}\right)$ in Equation (20) by chi-square fitting:

$$
\chi^{2}\left(t_{i}\right)=\sum_{j} \frac{\left\{I_{\mathrm{obs} j}\left(t_{i}\right)-F_{* j} R_{\mathrm{p}}^{2} \int_{s} \cos \theta_{0} \cos \theta_{1} d s \sum_{k} D_{j k}(\tau) A_{k}\left(t_{i}\right)\right\}^{2}}{\sigma_{j}^{2}\left(t_{i}\right)} .
$$

The fit is independently performed for the light curves at each epoch $t_{i}$. In order to quantify the errors of fitted values, we generate 100 realizations by adding a Poissonian error with 
rms of $\sigma_{j}$ to the simulated data, and compute the average and the variance of the best-fit values. We consider the photon shot noise alone in $\sigma_{j}$, ignoring the other statistical errors of the phase angle $\alpha$, star-planet distance $d$, and planet-observer distance $l$ among others. Thus our model is admittedly very idealized but indicates what one can learn from possible future data in principle.

Equation (26) is essentially a linear inverse problem. So as to ensure the positivity in each element of $A_{j}$, however, we replace $A_{j}$ by $B_{j}^{2}$ and search for the best-fit $B_{j}$ with a nonlinear fitting method (the Levenberg-Marquardt method, e.g., Press et al. 1992). We also made sure that another independent fitting method, which is based on a conditional least square method and does not use the above replacement (e.g., Menke 1989), gives very similar results.

Our model does not assume the condition $\sum_{k=1}^{4} A_{k}=1$. We could impose that constraint in principle, but it would somewhat restrict the applicability of the model. After all, the actual planetary surface does not exactly consist of only four Lambert surface types as we assumed here, and components other than the four types might contribute. In addition, the sum of the estimated area can easily deviate from unity due to a variety of other effects including the anisotropy of the scattering and the diversity of the detail features of the reflection spectra (e.g., the sharpness of the red edge, the slope of the soil spectrum etc.), Therefore, we fit the mock data without any constraints other than $A_{k}\left(t_{i}\right)>0$.

\subsection{Estimation of the Weighted Fractional Areas}

In reality, the atmospheric optical depth due to Rayleigh scattering $\tau$ will not be known in advance and should be fitted simultaneously from the data. In this subsection, however, we simply set the optical depth to the standard value $\tau_{0}$ :

$$
\tau_{0}(\lambda)=0.00864 \tilde{\lambda}^{-4}
$$

which is an approximation form of Equation (10) with $P=1013.25$ mbar. The effect of $\tau$ will be discussed in Section 3.5,

The top panel of Figure 8 shows the $\chi^{2} /$ d.o.f. (d.o.f. $=5-4=1$ ) of the fitting at each epoch. The second panel from the top presents the result of estimating weighted fractional areas $A_{k}\left(t_{i}\right)$ of ocean and land (= soil + vegetation + snow) using the light curves in bands $1,2,3,4$, and 5 . The third panel from the top panel displays the fractional areas of the three land types separately. The bottom panel illustrates the corresponding snapshot of the Earth toward the observer. The symbols indicate the average of the best-fit values

from the 100 realizations with quoted error bars representing the rms among them. For 
reference, we plot in dashed curves the weighted fractional areas based on the One-Minute Land Ecosystem Classification Product, which is generated from the official MODIS land ecosystem classification dataset classifying the surface of the Earth into 16 classes. Among the 16 classes, we regard "water" as ocean, "snow and ice" as snow, "open shrubland", "permanent wetlands", "urban and built-up", and "barren or sparsely vegetated" as soil, and others as vegetation, as shown in Table 2 .

The comparison of our best-fit values and dotted lines indicates that the present method works fairly well. Given the relatively crude approximation of the isotropic scattering and the assumption of only four surface types incorporated in the analysis, it is perhaps surprisingly successful. The presence of ocean, soil and vegetation is recovered, and the variation of their weighted fractional areas follows ground truth at least qualitatively. The bumps of the ocean at $t \sim 11$ and $20 \mathrm{hr}$ correspond to the Atlantic Ocean and the Pacific Ocean, respectively. The peaks in the soil and vegetation curves indeed correspond to the Sahara desert and the Amazon forest, respectively. Moreover, the weighted fractional area of snow is consistent with zero, in agreement with the fact that we have adopted the snow-free land BRDF data in the light curve simulation.

The quantitative discrepancy between the expected and model fractional areas of soil and of vegetation probably comes from the diversity of land scattering properties that is actually not well represented by the four Lambertian types shown in Figure 7. Note that our assignment of the 16 surface classification into the four types (Table 2) is not unique, and therefore the dashed lines should be regarded as a plausible but not unique reference.

Given the fact that scattered light from oceans comes almost exclusively from a very small region of specular reflection, it is perhaps puzzling that our inversion method, based on an assumption of Lambertian scattering, can estimate the fractional area of ocean reasonably well. This can be understood as follows: except for the specular reflection spot, the scattered light from the ocean surface is negligible (Figure 21). Rayleigh scattering in the atmosphere adds a uniform and very blue continuum to the observed flux from every part of the illuminated and visible part of the planetary surface, including areas of ocean away from the specular reflection point. For land areas, the contribution to the scattered light is dominated by direct scattering from the surface, especially in the redder bands, so the effect of Rayleigh scattering is merely to produce a slightly bluer overall color (Fig. (7). For ocean areas outside the specular reflection spot, however, the contribution to the total scattered light comes primarily from the Rayleigh scattering. Furthermore, the resulting scattered light by the atmosphere is fairly diffuse and comes from the entire illuminated and 
Table 2: The International Geosphere-Biosphere Programme(IGBP) classification which is generated from the official MODIS land ecosystem classification data-set(MOD12Q1). The fourth column is the merged classification we adopt for dashed lines in Figure 8 ,

\begin{tabular}{ccccc}
\hline \hline No. & IGBP Classification & Area(\%) & Our Classification & Ocean/Land \\
\hline 0 & water & 71.40 & ocean & ocean \\
1 & evergreen needleleaf forest & 1.13 & vegetation & land \\
2 & evergreen broadleaf forest & 2.87 & vegetation & land \\
3 & deciduous needleleaf forest & 0.18 & vegetation & land \\
4 & deciduous broadleaf forest & 0.46 & vegetation & land \\
5 & mixed forest & 1.34 & vegetation & land \\
6 & closed shrubland & 0.16 & vegetation & land \\
7 & open shrubland & 5.22 & soil & land \\
8 & woody savannas & 2.15 & vegetation & land \\
9 & savannas & 1.99 & vegetation & land \\
10 & grasslands & 2.65 & vegetation & land \\
11 & permanent wetlands & 0.06 & soil & land \\
12 & croplands & 2.56 & vegetation & land \\
13 & urban and built-up & 0.14 & soil & land \\
14 & cropland/natural vegetation mosaic & 0.60 & vegetation & land \\
15 & snow and ice & 3.16 & snow & land \\
16 & barren or sparsely vegetated & 3.92 & soil & land \\
17 & unclassified & 0.00 & - & - \\
\hline
\end{tabular}



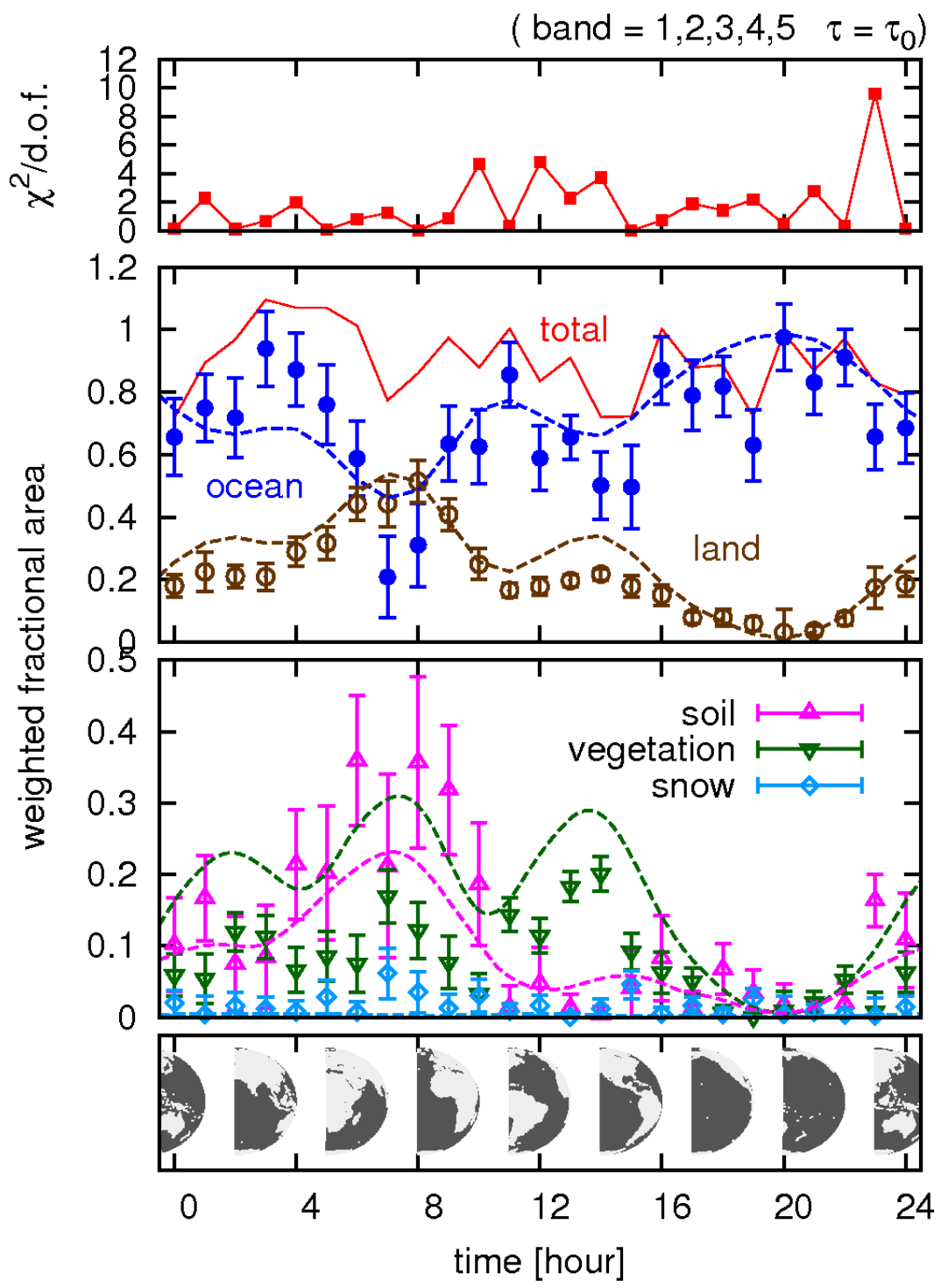

Fig. 8.- Reconstructed fractional areas $A_{k}\left(t_{i}\right)$ for four surface types from the simulated light curves in five bands (bands 1 - 5). The top panel shows the value of reduced $\chi^{2}$ $\left(=\chi^{2} /\right.$ dof where degree of freedom (dof) is $\left.5-4=1\right)$ for each epoch. The upper middle panel displays the results of estimating weighted fractional areas of ocean (blue), land (=soil+vegetation+snow; brown), and the total of them (red). The lower middle panel displays those of soil (magenta), vegetation (green), and snow (cyan). The dashed lines in those two panels show the weighted fractional areas derived from the MODIS land ecosystem classification dataset. The quoted error bars indicate the variance of the best-fit values from 100 realizations. The bottom panel depicts the snapshots of the Earth at the corresponding epochs where the ocean is painted in gray and the land in white. 
visible area. Thus, in order to account for the observed amount of Rayleigh scattering color in the total, the fit must assign a sufficiently large area of dark (very low albedo) area to the surface. Ocean is the only low albedo component available to the fit among the four components and thus is automatically assigned to produce enough Rayleigh scattering color without producing too much land scattering color. In other words, in our fit (which makes no use of spatial or time-domain information), the Rayleigh scattering component without any corresponding land component is a proxy for the ocean. The dark ocean surface is detectable in the fit to the colors primarily due to the additional Rayleigh scattering continuum supplied by the atmosphere above it.

\subsection{Time-integrated Spectra}

Since our fiducial observational condition (Table 1) is fairly idealized, the quoted errors are small even in the case of time-dependent analysis. In an actual missions, however, timeintergrated spectra will probably be the first realistic goal. The spectrum from the total integration time of the mock observation (2 weeks) is displayed in Figure 9. This result is obtained by adding up the photon counts for each band over two weeks and then normalizing them with respect to the scattered intensity of a lossless Lambert sphere. The error bars come from the shot noise of the total photon count in each band. Note that the red edge produces a sharp increase at $\lambda \sim 800 \mathrm{~nm}$.

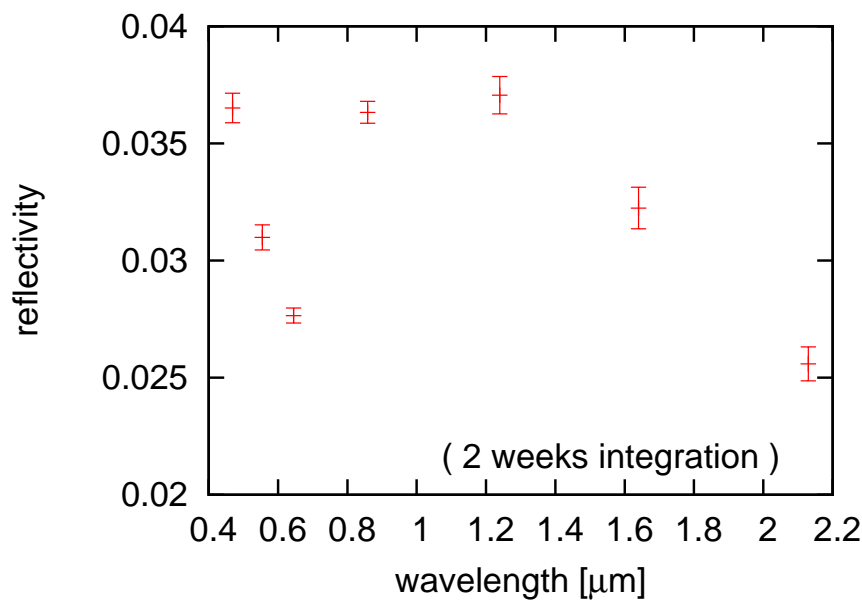

Fig. 9.- Spectrum of the cloudless Earth from the total integration time (2 weeks). The $y$-axis is normalized by the scattered light by a lossless Lambert sphere at full phase.

We present in Figure 10 the result of the fit to this time-averaged spectrum with our fiducial configuration. The fitting model is now the time-averaged version of Equation (20) 


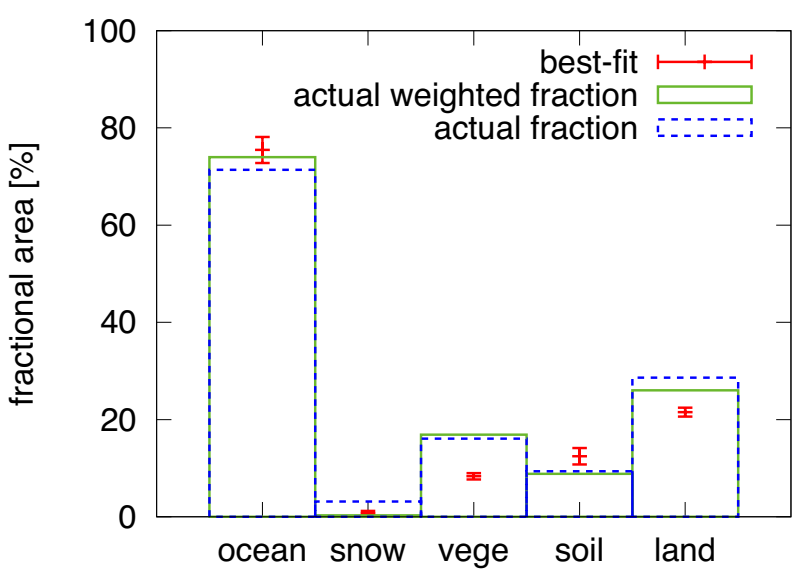

Fig. 10.- Time-averaged spectrum from the whole integration time (Figure 9) is decomposed into ocean, snow, vegetation, and soil with Equation (20). The land is the summation of snow, vegetation, and soil. The green boxes are the weighted fractional areas based on our merged classification (Table 22) and the blue boxes show the non-weighted actual fractions.

and thus the estimated values correspond to $\overline{A_{k}\left(t_{i}\right)}$ averaged over the full integration time. The blue boxes represent the actual fractional areas, while the green boxes represent the fractional areas based on our merged classification (Table 2) but correspondingly weighted to be compared with the estimated value. Under our fiducial configuration, the fractional areas are reasonably reproduced even without time resolution (and hence without spatial resolution).

We also repeat the same fitting procedure using mock light curves with different geometries, and summarize the results in Table 3. The values in the "reference" lines indicate weighted and averaged fractional areas visible for each observer. The bottom line shows the actual (non-weighted) fraction of the four surface components. Of course, the correspondence of the actual non-weighted fractional areas and the weighted fractional areas is highly dependent on the geometry. In the case of the Earth, the weighted fractions are roughly equivalent to the actual fractions in our fiducial configuration i.e. if the Earth is at equinox and the observer is on the equatorial plane, due to the fact that areas of ocean, soil, and vegetation are not extremely inhomogeneous but distributed well along the latitude. On the other hand, land areas would be preferentially seen as viewed by an observer located above the northern hemisphere. Although such geometric limitation is inevitable, the estimated values recover the weighted fractional areas for each geometry reasonably well. This is very encouraging for future missions. 
Table 3. Estimated values of weighted fractional areas from time-averaged spectra with different geometries. The "north $45^{\circ}$ " and "south $45^{\circ}$ " assumes the Earth at equinox seen with $+45^{\circ}$ and $-45^{\circ}$ inclination from the equatorial plane, respectively. The "summer (winter) solstice" assumes the Earth at summer (winter) solstice and the observer on the intersection of the equatorial plane and orbital plane. The values in "reference" lines are based on our merged classification shown in Table 2 which are weighted and averaged according to each geometry. The "actual (non-weighted)" is the non-weighted fractional areas of each components based on our merged classification.

\begin{tabular}{lllllll}
\hline \hline \multirow{2}{*}{ Geometry } & & ocean $(\%)$ & land $(\%)$ & soil $(\%)$ & vegetation $(\%)$ & snow $(\%)$ \\
\hline \multirow{2}{*}{ fiducial } & estimated & 75.5 & 21.5 & 12.4 & 8.3 & 0.7 \\
\cline { 2 - 7 } & reference & 74.0 & 26.0 & 8.8 & 16.8 & 0.3 \\
\hline \multirow{2}{*}{ north 45 } & estimated & 66.5 & 29.4 & 17.5 & 10.1 & 1.7 \\
\cline { 2 - 7 } & reference & 56.9 & 43.1 & 17.2 & 25.1 & 0.8 \\
\hline \multirow{2}{*}{ south 45 } & estimated & 86.6 & 10.7 & 4.3 & 5.6 & 0.7 \\
\cline { 2 - 7 } & reference & 85.6 & 14.3 & 4.0 & 6.9 & 3.4 \\
\hline \multirow{2}{*}{ summer solstice } & estimated & 73.1 & 26.5 & 16.3 & 9.4 & 0.8 \\
\cline { 2 - 7 } & reference & 68.0 & 32.3 & 11.5 & 20.3 & 0.03 \\
\hline \multirow{2}{*}{ winter solstice } & estimated & 83.7 & 16.6 & 9.3 & 7.3 & 0.1 \\
\cline { 2 - 7 } & reference & 79.2 & 20.8 & 6.3 & 13.1 & 1.4 \\
\hline actual (non-weighted) & 71.4 & 28.6 & 16.1 & 9.3 & 3.2 \\
\hline
\end{tabular}




\subsection{Estimation of the Optical Depth of Atmosphere}

So far we have assumed that the optical depth $\tau$ is indeed equivalent to the input value $\tau_{0}$. In reality, however, the value of $\tau$ would not be known a priori, and thus $\tau$ should be regarded as one of the fitting parameters. The use of an incorrect value of $\tau$ would degrade the fit. We investigate this issue by repeating the fit with different input optical depths:

$$
\tau_{\text {fit }}=\frac{n}{10} \tau_{0} \quad(n=0,1,2, \ldots, 20) .
$$

The matrix $D_{j k}$ depends on the value of $\tau$ (Equations (7), (13), (23), and (25)). Assuming that the optical depth is constant over the planetary surface, we sum up Equation (26) over different epochs according to

$$
\chi^{2}(\tau)=\sum_{i=1}^{24} \sum_{j=1}^{5} \frac{\left\{I_{\mathrm{obs} j}\left(t_{i}\right)-F_{* j} R_{\mathrm{p}}^{2} \int_{s} \cos \theta_{0} \cos \theta_{1} d s \sum_{k} D_{j k}(\tau) A_{k}\left(t_{i}\right)\right\}^{2}}{\sigma_{j}^{2}\left(t_{i}\right)},
$$

and search for the best-fit value of $\tau$ that minimizes Equation (29) among the different values (Equation (28) $)$.

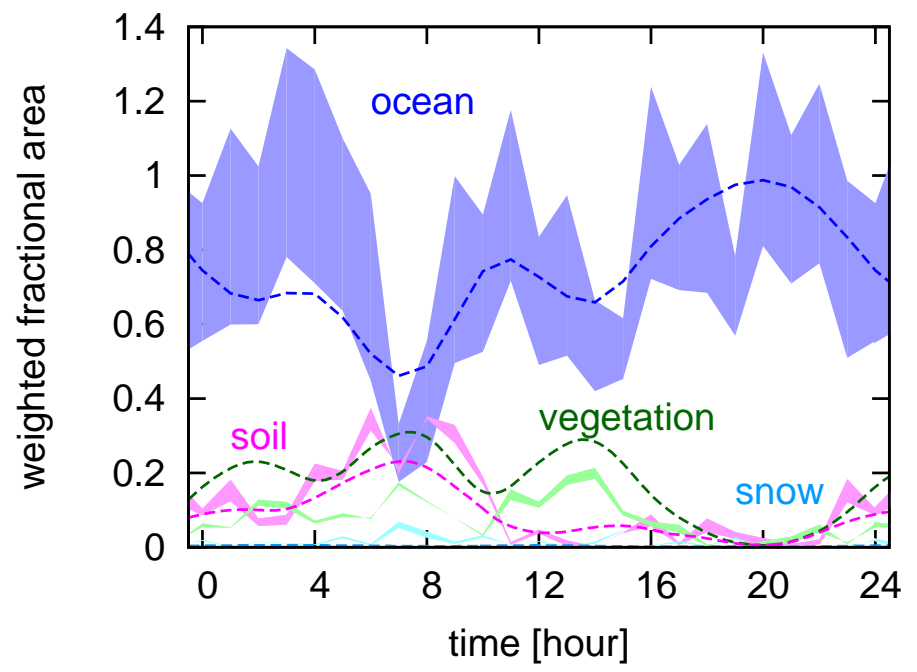

Fig. 11. - Dependence of reconstructed fractional areas on the input value of $\tau_{\text {fit }}$. The painted area exhibits the range of the estimated values when $\tau_{\text {fit }} / \tau_{0}$ varies from 0.5 to 1.5 . Simulated light curves in bands 1 to 5 are used. The dashed lines show the weighted fractional areas derived from the MODIS land ecosystem classification dataset, like those in Figure 8 .

It would be instructive to see first how the reconstructed fractional areas are sensitive to the value of $\tau_{\text {fit }}$. Figure 11 shows a case for which the simulated light curves with $\tau=\tau_{0}$ 
are fit using input values of $\tau_{\text {fit }}$ varied from $0.5 \tau_{0}$ to $1.5 \tau_{0}$. Since different values of $\tau_{\text {fit }}$ mainly modify the effective albedo of ocean through Rayleigh scattering, estimates of ocean fraction are sensitive to $\tau_{\text {fit }}$; ocean area is overestimated (underestimated) for small (large) $\tau_{\text {fit }}$. However, the estimate of the fractional areas for the other three surface types is fairly robust, indicating that these fractional areas are determined mainly at longer wavelengths where the value of $\tau$ does not make any significant difference.

Figure 12 displays the histogram of the best-fit values of $\tau_{\text {fit }}$ (Equation (28)) which minimize Equation (29). The results show a broad peak around $\tau_{\text {fit }} \sim 1.2 \tau_{0}$, but cases of $\tau_{\text {fit }}<0.9 \tau_{0}$ or $\tau_{\text {fit }}>1.6 \tau_{0}$ are relatively rare. Given the crude approximations adopted in our reconstruction method, these results are also encouraging.

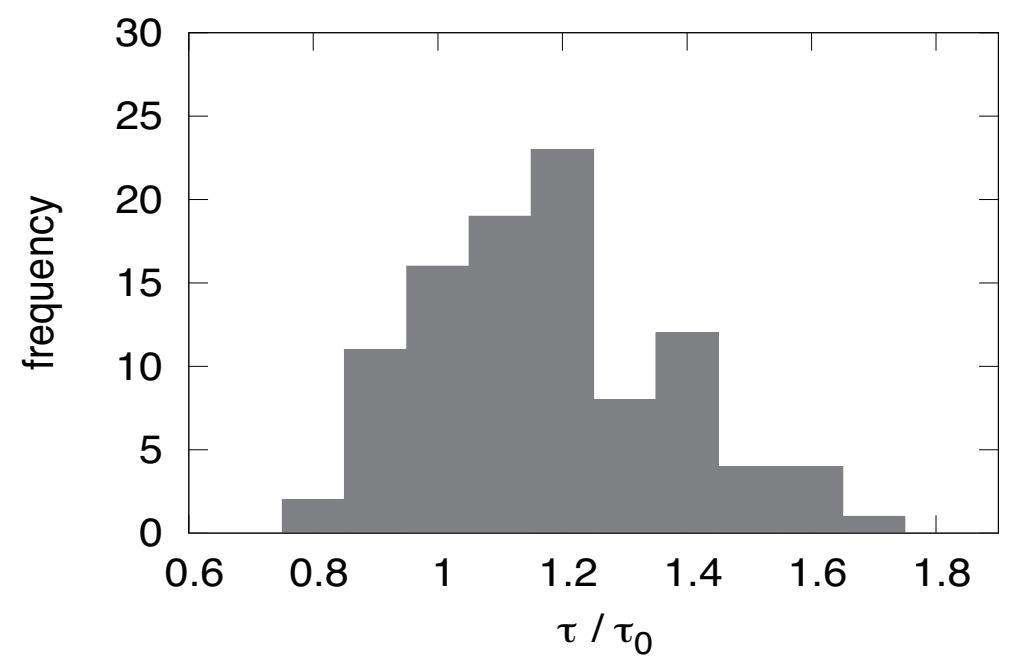

Fig. 12. - Histogram of best-fit values of $\tau_{\text {fit }}$ from 100 realizations of simulated light curves in bands 1 to 5 .

Thus we have found that our inversion method can recover the presence of ocean and atmosphere simultaneously for a cloudless Earth-like planet. It may be also instructive to consider a hypothetical ocean planet similar to Earth but without atmosphere at all. In this case the ocean contributes a tiny fraction of the total scattered light, and also a small fraction in terms of area because of the specular reflection.

In order to see this more quantitatively, we create light curves for the Earth without an atmosphere (i.e., Rayleigh scattering is neglected), and repeat the same analysis. The bestfit result with $\tau_{\text {fit }}=0$ in Equation (26) is shown in Figure 13. As expected, the estimate of the fractional areas of ocean is very unstable and unreliable. It is interesting to note, however, that one can still reconstruct the fractional areas of soil and vegetation fairly well. 


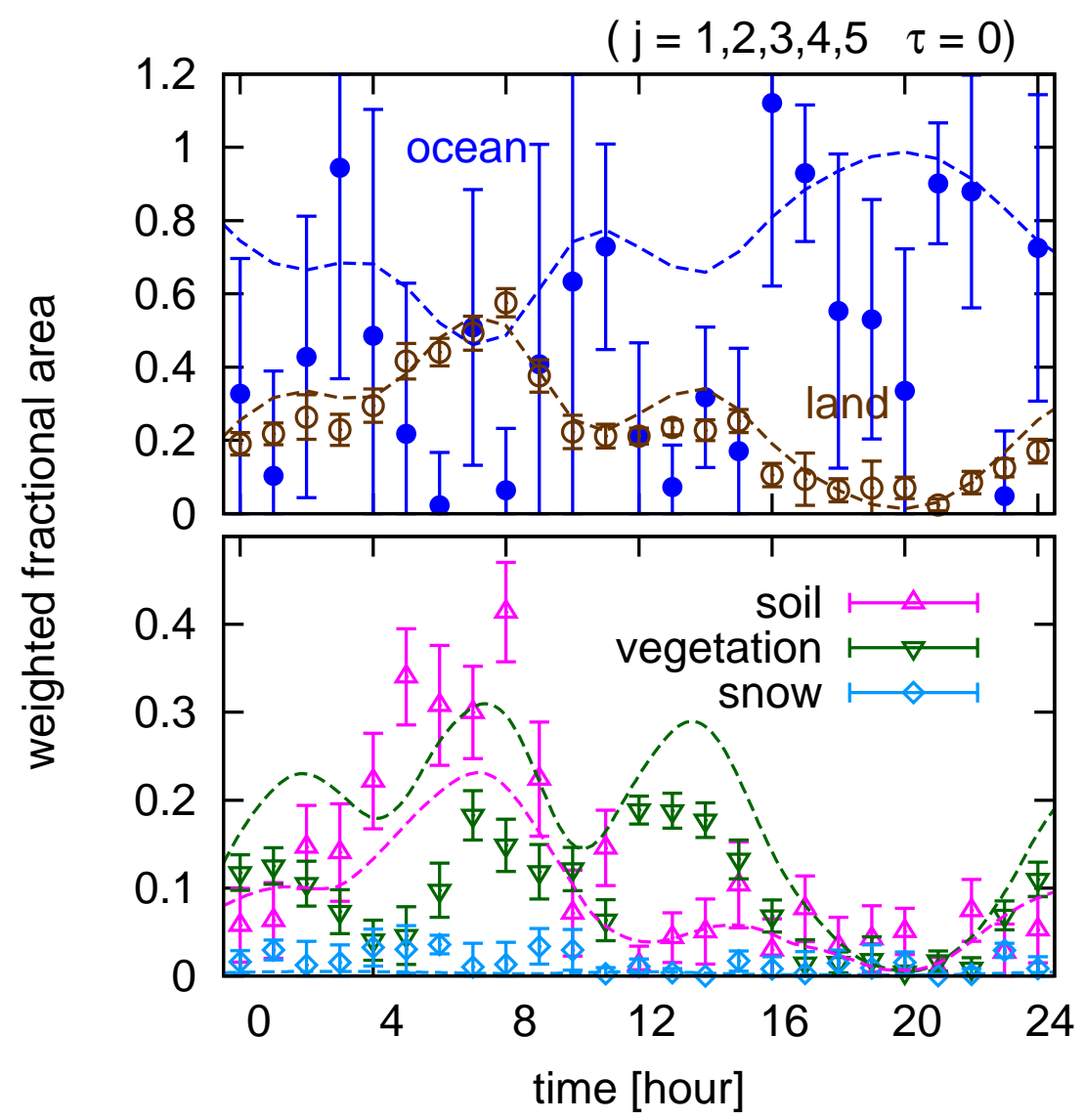

Fig. 13.- Same as Figure 8 but without an atmosphere.

\section{Discussion}

\subsection{Band Selection}

Our inversion method relies entirely on the difference of the wavelength-dependence of the scattering spectra among ocean, soil, vegetation, and snow, or their colors in short. Therefore the selection of the observed bands is crucial.

First we repeat the same analysis performed in Section 3 but with four bands. In the case of five bands, we can fit up to five unknowns; we selected fractional areas for the four types and $\tau$ as the five fitting parameters. In the case of 4 bands, however, we cannot fit $\tau$ simultaneously and thus fix $\tau=\tau_{0}$. The result is shown in Figure 14, The left and right 
panels correspond to the bluer bands $(j=1,2,3$, and 4$)$ and redder bands $(j=4,5,6$, and 7 ), respectively. The difference can be easily understood; the bluer bands are more sensitive to the effect of Rayleigh scattering and the fractional area of ocean is recovered fairly well. Also the red edge feature between bands 3 and 4 still carries the vegetation signature, while the fractional area of soil becomes more uncertain because it is brighter in the redder bands. The result with the redder bands shows consistently opposite generic features; the lack of the blue bands and the red edge makes it difficult to detect the signature of ocean and vegetation, respectively, while the soil is more easily reconstructed.
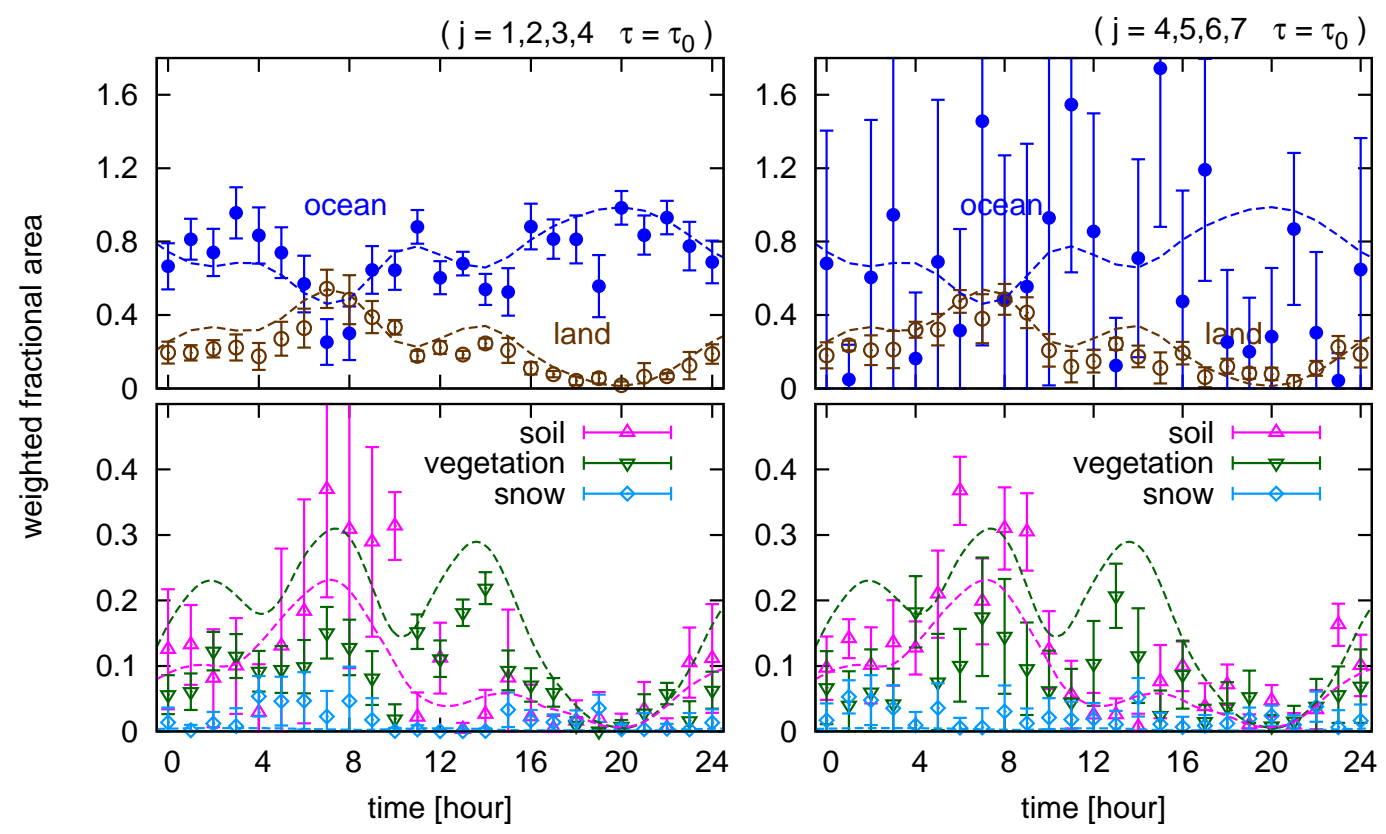

Fig. 14. - Reconstructed fractional areas using simulated light curves in two different sets of four bands; Left: bluer four bands $(j=1,2,3$, and 4$)$, Right: redder four bands $(j=4$, $5,6$, and 7$)$.

A closer look at Figure 14 indicates some degeneracy between reflection spectra of selected surface types (Figure 7). This is more clearly illustrated in Figure 15, where we use three bands $(j=2,3$, and 4$)$ only. In this case we cannot determine four surface types, and we choose ocean, soil, and vegetation in the left panel, and ocean, vegetation, and snow in the right panel, while we neglect the remaining surface type from the fit. The result naturally is degraded compared with the 4-band and 5-band cases. The vegetation signature is still there because we chose bands 3 and 4 that bracket the red edge, and the fractional areas of soil and snow compensate for each other. The fact that the spectra of snow and 
soil are similar in shorter wavelength bands (except for their amplitude) partly explains the behavior of the lower panels in Figure 15. While this similarity may come largely from our single scattering approximation as described in Section 3.1, we obtained a similar result even when we use the snow effective albedo of $\tau=0$. Therefore the degeneracy between snow and soil is fairly generic as long as we use the short wavelength bands in the reconstruction.
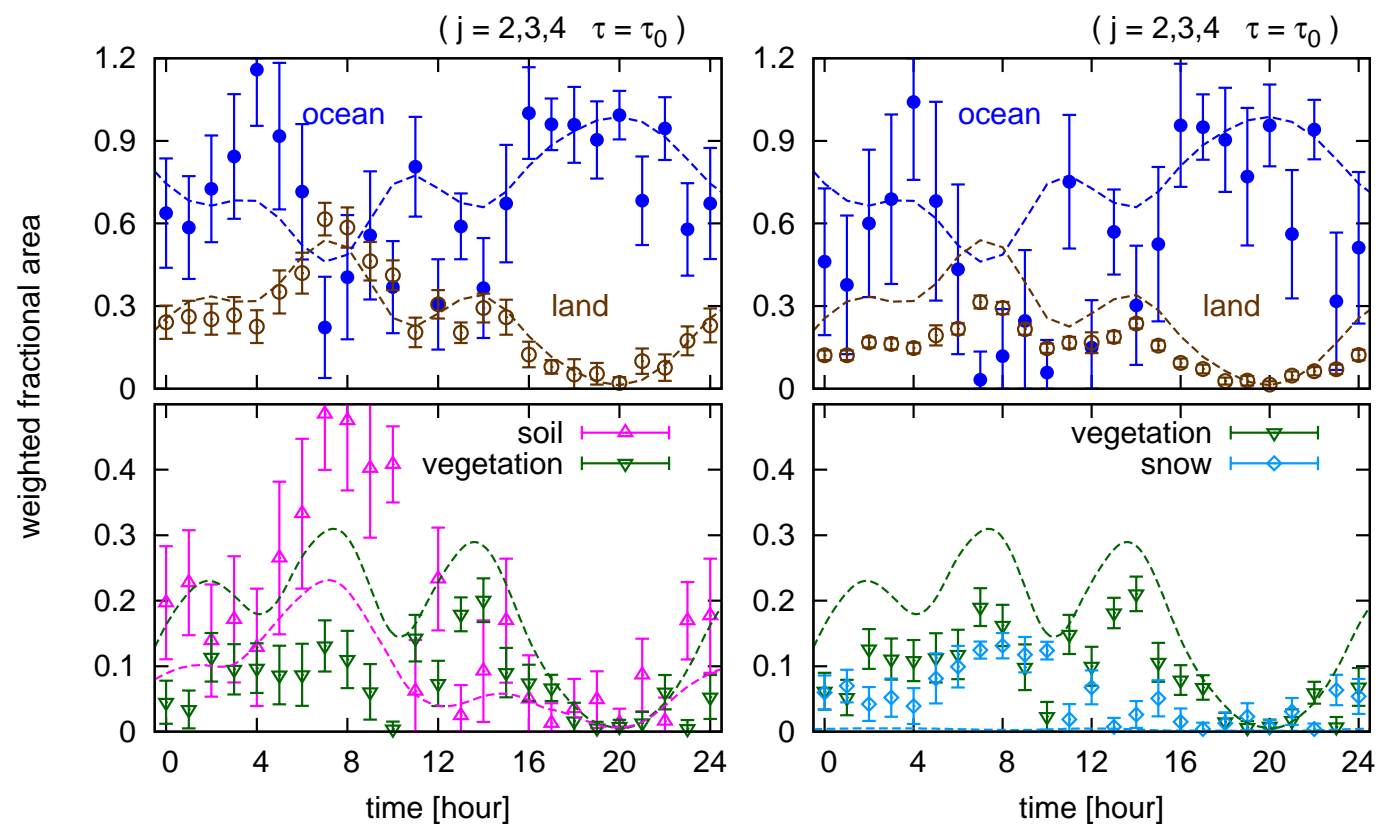

Fig. 15.- Reconstructed fractional areas using simulated light curves in three bands; Left: ocean, soil, and vegetation are considered. Right: ocean, vegetation, and snow are considered.

\subsection{Principal Component Analysis (PCA)}

Cowan et al. (2009) performed a PCA of the multi-band light curves of the Earth as observed by the EPOXI mission. They extracted two major eigenspectra in a modelindependent manner. We also performed PCA on the same data-set, and obtained results consistent with those of Cowan et al. (2009). We then performed PCA of our mock light curves and again extracted two major eigenspectra; these are shown in Figure 16.

In order to compare our current methodology with PCA, we decompose these two eigenspectra into a combination of the effective albedo of the four surface types with $\tau=\tau_{0}$ 
(solid lines in Figure 7). We find that the first eigenspectrum roughly corresponds to (soil+vegetation-ocean), and the second one roughly corresponds to (vegetation-(soil+snow+ocean)) as displayed in Figure 17.

This result indicates that the extracted eigenspectra do not necessarily correspond to any single surface type. This is not surprising, of course. While PCA extracts orthogonal eigenspectra by definition, the wavelength dependence of albedos of real materials is not orthogonal in general. Moreover, they are likely to be degenerate in PCA because the fractional areas are complementary; for example, the fractional area of ocean decreases when fractional area of land increases. In other words, the time dependences of these components are necessarily correlated. Therefore, it seems natural that the first eigenspectrum is roughly (land-ocean). Our method is quite model dependent, but it allows decomposition of the light curves into physical components that can be interpreted in a simple way. While the model independence of PCA is a great advantage, the final interpretation is not straightforward. Further comparison with PCA is beyond the scope of this paper, but clearly these two methodologies are very complementary.
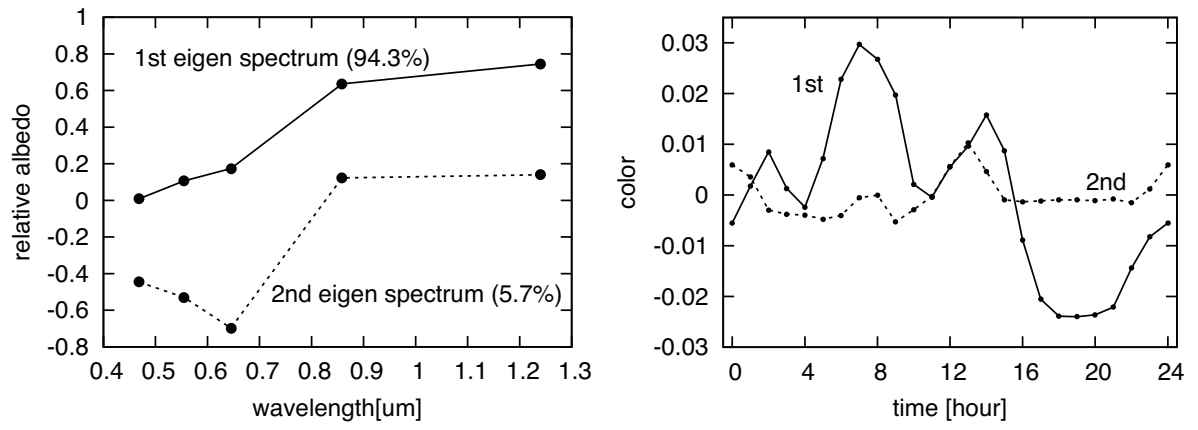

Fig. 16. - Left: the eigenspectra extracted by PCA of our fiducial mock light curves displayed in Figure 3. The contribution of the first eigenspectrum (solid) is $94.3 \%$ and that of the second one (dashed) is $5.7 \%$. Right: the time variation of the first eigenspectrum (solid) and the second eigenspectrum (dashed).

\section{Summary}

In this paper we have presented a method to reconstruct the fractional areas of different surface components from photometric colors of Earth-like exoplanets without clouds. For this purpose we first created mock light curves in seven photometric bands from the observed data of the Earth, but neglected the clouds. The light curves are fitted to isotropic scat- 

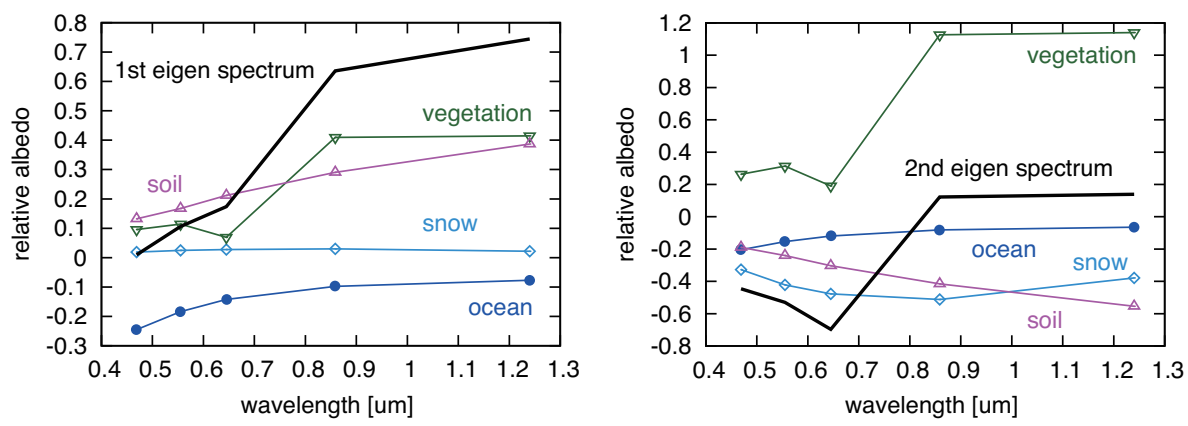

Fig. 17. - Left: decomposition of the first eigenspectrum of Figure 16] where the coefficients are ocean, -2.19; snow, 0.03; vegetation, 0.86; soil, 1.07. Effective albedos with $\tau=\tau_{0}$ are used. Right: decomposition of the second eigenspectrum of Figure 16 where the coefficients are ocean, -1.83; snow, -0.58; vegetation, 2.36; soil, -1.54. Effective albedos with $\tau=\tau_{0}$ are used.

tering models consisting of four surface types: ocean, soil, snow, and vegetation. In a very idealized situation where the data are obtained using a noiseless $2 \mathrm{~m}$ telescope and multiple integrations of $1 \mathrm{hr}$ each, we find that our method is able to reproduce the fractional areas of those components fairly well. In particular, Figures 8, 11, 13, 14, and 15, show quantitatively that the presence of vegetation can be recovered using the color information via its red edge feature. Although we fit the fractional areas of each component independently at each time in the light curves, the time variation is eventually translated into the distribution along the longitude with the methodology described in Cowan \& Agol (2008).

We also find that Rayleigh scattering due to the atmosphere plays a key role in estimating reliably the fractional areas of ocean. Indeed, without an atmosphere, our method based on the isotropic scattering assumption cannot properly estimate the real fractional area of ocean because of the strongly anisotropic nature of its specular reflection. On the other hand, for terrestrial exoplanets with atmosphere similar to our Earth, we may be able to estimate the presence of ocean and atmosphere simultaneously if the effect of clouds is safely neglected.

Our methodology described in this paper is based on admittedly several very idealized assumptions and simplifications. There are a variety of issues that we have to address and improve in future work, some of which are listed below.

First, one of the most serious omissions in the present modeling is the absence of clouds. Clouds provide additional time variation in the light curves, which is not directly related to the property of the planetary surface. Clouds greatly increase the reflectivity in visible and 
near-infrared bands. We may treat clouds as an additional component in our current model. While the time and spatial variabilities of clouds are not easy to model, combining data from multiple rotation periods may allow us to separate variations due to surface features from those caused by clouds. We will discuss these effects of clouds more quantitatively in future work.

Second, the universality of the scattering spectra of surface types on Earth is not clear, maybe not even likely. Indeed this could be regarded as both the strongest and the weakest point in our methodology; we can recover surface information, including the presence of vegetation from the color variations alone because our spectral template is derived from that of the Earth. Nevertheless, as stressed in the introduction, it is an important first attempt to see if we could infer the presence of vegetation observationally for exoplanets similar to the Earth even in the most favorable and idealized circumstances. The answer to the question seems promising and the next step is to generalize the result for a wider range of possibilities. The scattering properties of ocean and pure snow may be universal because the properties of $\mathrm{H}_{2} \mathrm{O}$ would not be different on different planets, but they can be somewhat different depending on impurities, for example. The generality of the red edge depends on the photosynthesis system of exo-vegetation and the wavelength of that "edge" is likely to shift according to the spectral type of the host star (Wolstencroft \& Raven 2002; Kiang et al. $2007 \mathrm{a}, \mathrm{b})$. Also, the scattering properties of soil may vary depending on the details of the soil's composition, and even the gravity may affect the granularity which changes the scattering properties.

Finally we can explore other geometrical configurations of a star-planet system including the effects of orbital inclination, planet obliquity, and seasonal variations. It will be interesting to apply our methodology to time-series remote sensing data-sets for the Earth as well as to models of the Earth in the distant past which take into account continental drift and the evolutionary history of vegetation.

Y.F. and Y.S. gratefully acknowledge support from the Global Collaborative Research Fund (GCRF) "A World-wide Investigation of Other Worlds" grant and the Global Scholars Program of Princeton University, respectively. They also thank people in Peyton Hall, Princeton University, where this paper was completed, for their warm hospitality and discussions. In particular, we are grateful to Adam Burrows for his helpful comments on this paper. H.K. is supported by JSPS (Japan Society for the Promotion of Science) Fellowship for Research (DC2:20-10466), A.T. by a JSPS grant (No.21740168). E.L.T is also supported in part by the aforesaid GCRF grant and the World Premier International Research Center Initiative (WPI Initiative), Ministry of Education, Culture, Sports, Science and Technology, Japan. This work is also supported by JSPS Core-to-Core Program "International Research 
Network for Dark Energy."

\section{A. BRDF for land}

We create mock light curves adopting the Rossi-Li model (Equation (14)) for the land BRDF. The specific expressions for the volume-scattering term, $K_{\mathrm{vol}}$, and the geometricoptical term, $K_{\text {geo }}$, are given here for completeness. The derivation of the two terms is found in Wanner et al. (1995). The volume-scattering term is

$$
K_{\mathrm{vol}}\left(\theta_{0}, \theta_{1}, \phi\right)=\frac{(\pi / 2-\xi)+\sin \xi}{\cos \theta_{0}+\cos \theta_{1}}-\frac{\pi}{4},
$$

where

$$
\cos \xi=\cos \theta_{0} \cos \theta_{1}+\sin \theta_{0} \sin \theta_{1} \cos \phi .
$$

The geometric-optical term is:

$$
K_{\text {geo }}\left(\theta_{0}, \theta_{1}, \phi\right)=O\left(\theta_{0}, \theta_{1}, \phi\right)-\sec \theta_{0}^{\prime}-\sec \theta_{1}^{\prime}+\frac{1}{2}\left(1+\cos \xi^{\prime}\right) \sec \theta_{0}^{\prime} \sec \theta_{1}^{\prime},
$$

where

$$
\begin{aligned}
O\left(\theta_{0}, \theta_{1}, \phi\right) & =\frac{1}{\pi}(t-\sin t \cos t)\left(\sec \theta_{0}^{\prime}+\sec \theta_{1}^{\prime}\right), \\
\cos t & =\min \left\{1, \frac{h}{b} \frac{\sqrt{D^{2}+\left(\tan \theta_{0}^{\prime} \tan \theta_{1}^{\prime} \sin \phi\right)^{2}}}{\sec \theta_{0}^{\prime}+\sec \theta_{1}^{\prime}}\right\} \\
D & =\sqrt{\tan ^{2} \theta_{0}^{\prime}+\tan ^{2} \theta_{1}^{\prime}-2 \tan \theta_{0}^{\prime} \tan \theta_{1}^{\prime} \cos \phi} \\
\cos \xi^{\prime} & =\cos \theta_{0}^{\prime} \cos \theta_{1}^{\prime}+\sin \theta_{0}^{\prime} \sin \theta_{1}^{\prime} \cos \phi \\
\theta_{0}^{\prime} & =\tan ^{-1}\left(\frac{b}{r} \tan \theta_{0}\right) \\
\theta_{1}^{\prime} & =\tan ^{-1}\left(\frac{b}{r} \tan \theta_{1}\right) .
\end{aligned}
$$

This kernel assumes a sparse ensemble of surface objects which throws shadows on the Lambertian background. The objects are approximated as spheroids with width $2 r$ and vertical length $2 b$. The height of the center of the spheroids is denoted by $h$. The function $O\left(\theta_{0}, \theta_{1}, \phi\right)$ is the overlap area between the solar shadows and the shade of view. For MODIS processing $h / b=2$ and $b / r=1$ are assumed (i.e., the spherical crowns are separated from the ground by their radii). Thus, we adopted these values. 


\section{B. BRDF for ocean}

The BRDF model for ocean is very complicated, and we summarize the key expressions of the model by Nakajima \& Tanaka (1983) that we adopt in the present work.

The scattering of solar radiation by a flat ocean follows the simple Snell-Fresnel law. Using the pair $\{\theta, \phi\}$ to represent polar coordinates relative to the normal of the averaged

surface plane (Figure 18), the relation between the incident radiance $I_{\text {in }}\left(\theta^{\prime}, \phi^{\prime}\right)$ and the scattered radiance $I_{\text {out }}(\theta, \phi)$ is written as

$$
\begin{aligned}
I_{\text {out }}(\theta, \phi) & =\int d \cos \theta^{\prime} d \phi^{\prime} R\left(\theta, \theta^{\prime}, \phi-\phi^{\prime}\right) I_{\text {in }}\left(\theta^{\prime}, \phi^{\prime}\right), \\
R\left(\theta, \theta^{\prime}, \phi-\phi^{\prime}\right) & =r(\theta, \tilde{m}) \delta\left(\cos \theta-\cos \theta^{\prime}\right) \delta\left(\phi^{\prime}-\phi-\pi\right),
\end{aligned}
$$

where $r(\theta, \tilde{m})$ is the Fresnel scattering coefficient and $\tilde{m}$ the is the ratio of the reflective indices of air and ocean. When the wind above ocean is taken into account, however, the slope of scattering surface varies both temporally and spatially.

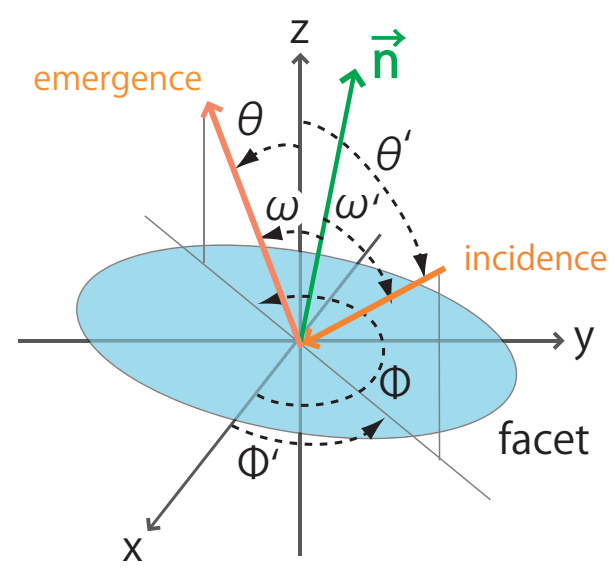

Fig. 18. - Configuration of incident and scattered rays with respect to a wave facet whose normal vector $\mathbf{n}$ is $\left\{\theta_{n}, \phi_{n}\right\}$ in the polar coordinates with respect to the average surface plane.

Thus, the normal direction of the facet is different from that of the averaged surface plane (z-axis in Figure [18). We introduce the polar coordinate $\{\omega, \xi\}$ with respect to the normal vector $\mathbf{n}$ of each facet as shown in Figure 18, Then the scattered radiance including the effect of rough surface is written as

$$
\begin{aligned}
& I_{\text {out }}(\theta, \phi)= \frac{1}{\cos \theta} \int d \cos \theta_{n} \int d \phi_{n} \int d \cos \theta^{\prime} \int d \phi^{\prime} \cos \omega \\
& \times S\left(\theta, \theta^{\prime}, \phi-\phi^{\prime}, \theta_{n}, \phi_{n}\right) R\left(\omega, \omega^{\prime}, \xi-\xi^{\prime}\right) I_{\text {in }}\left(\theta^{\prime}, \phi^{\prime}\right) .
\end{aligned}
$$


In the above, $\left\{\theta_{n}, \phi_{n}\right\}$ denotes the direction of the normal of the facet, and $S$ is the effective fractional area of the wave facet associated with $\left\{\theta_{n}, \phi_{n}\right\}$. The integration over $\theta_{n}$ and $\phi_{n}$ results in setting $\omega=\omega^{\prime}$ due to the Dirac delta in Equation (B2). Then Equation (B1) reduces to

$$
\begin{aligned}
& I_{\text {out }}(\theta, \phi)=\int d \cos \theta^{\prime} \int d \phi^{\prime}\left|\frac{\partial\left(\cos \theta_{n}, \phi_{n}\right)}{\partial\left(\cos \theta^{\prime}, \phi^{\prime}\right)}\right| \cos \omega^{*} \\
& \times S\left(\theta, \theta^{\prime}, \phi-\phi^{\prime}, \theta_{n}^{*}, \phi_{n}^{*}\right) r\left(\omega^{*}, \tilde{m}\right) I_{\text {in }}\left(\theta^{\prime}, \phi^{\prime}\right),
\end{aligned}
$$

where $\theta_{n}^{*}, \phi_{n}^{*}$, and $\omega^{*}$ are defined through the conditions of $\omega=\omega^{\prime}$ and $\xi-\xi^{\prime}=\pi$, or more explicitly,

$$
\begin{aligned}
\cos \theta_{n}^{*} & =\frac{\cos \theta+\cos \theta^{\prime}}{2 \cos \omega^{*}} \\
\cos \left(2 \omega^{*}\right) & =\cos \theta \cos \theta^{\prime}+\sin \theta \sin \theta^{\prime} \cos \left(\phi-\phi^{\prime}\right)
\end{aligned}
$$

as shown in Figure 18, The Jacobian determinant in Equation (B4) is

$$
\left|\frac{\partial\left(\cos \theta_{n}, \phi_{n}\right)}{\partial\left(\cos \theta^{\prime}, \phi^{\prime}\right)}\right|=\frac{1}{4 \cos \omega^{*}}
$$

The effective fractional area $S$ is expressed as

$$
\Sigma S d \cos \theta_{n} d \phi_{n}=\frac{\Sigma}{\cos \theta_{n}} P\left(\theta_{n}, \phi_{n}\right) \mathcal{T}\left(\theta_{0}, \theta_{1}^{\prime} ; \phi, \phi^{\prime} \mid \theta_{n}, \phi_{n}\right) d \cos \theta_{n} d \phi_{n},
$$

where $\Sigma$ is the horizontal area, $P$ is the density function of the wave slope, and $\mathcal{T}$ is the bidirectional shadowing factor. The factor $P\left(\theta_{n}, \phi_{n}\right)$ is empirically given (see Figure 3 of Nakajima \& Tanaka 1983) by

$$
P\left(\theta_{n}, \phi_{n}\right)=\frac{1}{\pi \sigma^{2} \cos ^{3} \theta_{n}} \exp \left(-\frac{\tan ^{2} \theta_{n}}{\sigma^{2}}\right) \equiv p\left(\theta_{n}\right) .
$$

Here, $\sigma$ is the rms of slopes and a function of the wind velocity, $u_{10}$, at $10 \mathrm{~m}$ height above the water surface taken to be

$$
\sigma^{2}=0.00534 u_{10}
$$

Another factor, $\mathcal{T}\left(\theta, \theta^{\prime} ; \phi, \phi^{\prime} ; \theta_{n}, \phi_{n}\right)$, is obtained from an analytical fit to the results of a Monte Carlo simulation assuming one-dimensional random surface with Gaussian autocorrelation, which resulted in

$$
\begin{aligned}
\mathcal{T}\left(\theta, \theta^{\prime} ; \phi, \phi^{\prime} \mid \theta_{n}, \phi_{n}\right) & =H\left(v-\frac{\partial z / \partial x}{\sigma}\right) H\left(v^{\prime}-\frac{\partial z / \partial x}{\sigma}\right) G\left(v, v^{\prime}\right) \\
v & =\frac{\cos \theta}{\sigma \sin \theta}, \quad v^{\prime}=\frac{\cos \theta^{\prime}}{\sigma \sin \theta^{\prime}}
\end{aligned}
$$




$$
\begin{aligned}
G\left(v, v^{\prime}\right) & =\frac{1}{1+F(v)+F\left(v^{\prime}\right)} \\
F(v) & =\frac{1}{2}\left[\frac{\exp \left(-v^{2}\right)}{\sqrt{\pi} v^{2}}-\frac{2}{\sqrt{\pi}} \int_{v}^{\infty} \exp \left(-t^{2}\right) d t\right]
\end{aligned}
$$

where $\partial z / \partial x$ is the slope of the facet, $H(x)$ is the Heaviside step function, and $\sigma$ is given by Equation (B10).

Combining all the expressions above, Equation (B4) is now written as:

$$
\begin{aligned}
I_{\text {out }}(\theta, \phi) & =\int d \cos \theta^{\prime} \int d \phi R\left(\theta, \theta^{\prime}, \phi-\phi^{\prime}\right) I_{\text {in }}\left(\theta^{\prime}, \phi^{\prime}\right), \\
R\left(\theta, \theta^{\prime}, \phi-\phi^{\prime}\right) & =\frac{1}{\cos \theta \cos \theta_{n}^{*}} p\left(\theta_{n}^{*}\right) G\left(v, v^{\prime}\right) r\left(\omega^{*}, \tilde{m}\right),
\end{aligned}
$$

where $\theta_{n}^{*}$ and $\omega^{*}$ are functions of $\theta, \theta^{\prime}$, and $\left(\phi-\phi^{\prime}\right)$ through Equations (B5) and (B6).

Considering a parallel incident flux $F_{*}$ from the direction of $\left\{\theta_{0}, \phi_{0}\right\}$, we have

$$
I_{\text {in }}(\theta, \phi)=F_{*} \delta\left(\cos \theta^{\prime}-\cos \theta_{0}\right) \delta\left(\phi^{\prime}-\phi_{0}\right)
$$

Substituting Equation (B17) into Equation (B15) and replacing $\{\theta, \phi\}$ with $\left\{\theta_{1}, \phi_{1}\right\}$, we finally arrive at

$$
\begin{aligned}
I_{\text {out }}\left(\theta_{1}, \phi_{1}\right) & =F_{*} R\left(\theta_{0}, \theta_{1}, \phi_{0}-\phi_{1}\right) \\
R\left(\theta_{0}, \theta_{1}, \phi_{1}-\phi_{0}\right) & =\frac{1}{\cos \theta_{1} \cos \theta_{n}^{*}} p\left(\theta_{n}^{*}\right) G\left(v, v^{\prime}\right) r\left(\omega^{*}, \tilde{m}\right) .
\end{aligned}
$$

This is equivalent to Equation (15) in Section 2.2.4:

$$
f_{\text {ocean }}\left(\theta_{0}, \theta_{1}, \phi\right)=\frac{1}{4 \cos \theta_{0} \cos \theta_{1} \cos \theta_{n}^{*}} p\left(\theta_{n}^{*}\right) G\left(v, v^{\prime}\right) r\left(\omega^{*}, \tilde{m}\right) .
$$

\section{REFERENCES}

Arnold L., Gillet S., Lardire O., Riaud P., \& Schneider J. 2002, A\&A, 392, 231

Baldridge, A. M., Hook, S. J., Grove, C. I., \& Rivera, G. 2009, Remote Sens. Environ., 113, 711

Ballester, G. E., Sing, D. K. \& Herbert, F. 2007, Nature, 445, 511

Bennett, D. P., et al. 2008, ApJ, 684, 663 
Charbonneau, D., Brown, T. M., Noyes, R. W. \& Gilliland, R. L. 2002, ApJ, 568, 377

Cowan, N. B., \& Agol, E. 2008, ApJ, 678, L129

Cowan, N. B., Agol, E., Meadows, V. S. \& Robinson, T. 2009, ApJ, 700, 915

ESA SCI-A, 2007, Darwin Mission Summary Status Issue 2.0 (Noordwijk: ESA)

Des Marais, D. J., et al. 2002, Astrobiology, 2, 153

Ford, E., Seager, S. \& Turner, E. L. 2001, Nature, 412, 885

Frölich, C., \& Shaw, G. E. 1980, Appl. Opt., 19, 1773

Goode, P. R., Qiu, J., Yurchyshyn, V., Hickey, J., Chu, M-C, Kolbe, E., Brown, C. T. \& Koonin, S. E. 2001, J. Geophys. Res. Lett., 28, 1671

Kasdin, N. J., et al., 2010, BAAS, 41, 287

Kasting, J. F., \& Catling, D. 2003, ARA\&A, 41, 429

Kasting, J. F., Whitmire, D. P. \& Raynolds, R. T. 1993, Icarus, 101, 108

Kiang, N. Y., Siefert, J., Govindjee, Blankenhship, R. E. 2007, Astrobiology, 7, 222

Kiang, N. Y., et al., 2007, Astrobiology, 7, 252

Lawson, P. R., et al. 2009, in Astro2010 — The Astronomy and Astrophys. Decadal Survey, 53

Levine, M., et al. 2009, "Terrestrial Planet Finder - Coronagraph (TPF-C) Flight Baseline Mission Concept", arXiv:0911.3200

Leger, A., Pirre, M., \& Marceau, F. J. 1993, A\&A, 277, 309

Lucht, W., Schaaf, C. \& Strahler, A. H. 2002, IEEE Trans. Geosci. Remote Sens., 38, 977

Mayor, M., et al. 2009, A\&A, 493, 639

Menke, W. 1989, Geophysical Data Analysis: Discrete Inverse Theory (Revised ed.; New York: Academic)

Montañés-Rodriguéz, P., Pallé, E., Goode, P. R. \& Martín-Torres, F. J. 2006, ApJ, 651, 544

Nakajima, T. \& Tanaka, M. 1983, M. Quant. Spectrosc. Radiat. Transfer, 29, 521 
Oakley, P. H. H. \& Cash, W. 2009, ApJ, 700, 1428

Pallé, E., Ford, E. B., Seager, S., Montanés-Rodríguez, P. \& Vazquez, M. 2008, ApJ, 676, 1319

Press, W. H., Flannery, B. P., Teukolsky, S. A. \& Vetterling, W. T. 1992, Numerical Recipes in C: The Art of Scientific Computing (Cambridge: Cambridge Univ. Press)

Queloz, D., et al. 2009, A\&A, 506, 303

Salomonson, V. V., et al. 1989, IEEE Trans. Geosci. Remote Sensing, 27, 145

Seager, S., Turner, E. L., Schafer, J. \& Ford, E. B., 2005, Astrobiology, 5, 372

Swain, M. R., Vasisht, G. \& Tinetti, G. 2008, Nature, 452, 20

Swain, M. R., Vasisht, G., Tinetti, G., Bouwman, J., Chen, P., Yung, Y., Deming, D., \& Deroo, P. 2009, ApJ, 690, L114

Tinetti, G., Meadows, V. S., Crisp, D., Fong, W., Fishbein, E., Turnbull, M. \& Bibring, J.-P. 2006, Astrobiology, 6, 34

Tinetti, G., Meadows, V. S., Crisp, D., Kiang, N. Y., Kahn, B. H., Fishbein, E., Velusamy, T. \& Turnbull, M. 2006, Astrobiology, 6, 881

Tinetti, G., et al. 2007, Nature, 448, 169

Vidal-Madjar, A., Lecavelier des Etangs, A., Désert, J.-M., Ballester, G. E., Ferlet, R., Hébrard, G. \& Mayor, M. 2003, Nature, 422, 143

Vidal-Madjar, A., et al. 2004, ApJ, 604, 69

Wanner, W., Li, X. \& Strahler, A. H. 1995, J. Geophys. Res. Lett., 100, 21077

Williams, D. M. \& Gaidos, E. 2008, Icarus, 195, 927

Wolstencroft, R. D. \& Raven, J. A. 2002, Icarus, 157, 535

Woolf, N. J., Smith, P. S., Traub, W. A. \& Jucks, K. W. 2002, A\&A, 547, 430

Young, A. T. 1980, Appl. Opt., 19, 3427 